\title{
Purchasing, production, and sales strategies for a production system with limited capacity and fluctuating sales and purchasing prices*
}

\begin{abstract}
Oktay Karabă̆
College of Engineering, Koç University, Rumeli Feneri Yolu, Istanbul, Turkey, 34450,

Email: okarabag13@ku.edu.tr Phone: +90212 3381348 Fax: +90 2123381395

Barış Tan

College of Administrative Sciences and Economics, Koç University, Rumeli Feneri Yolu, Istanbul, Turkey, 34450, Email: btan@ku.edu.tr Phone: +90 2123381459 Fax: +90 2123381653

In many industries, the revenue and cost structures of manufacturers are directly affected by the volatility of purchasing and sales prices in the markets. We analyze the purchasing, production, and sales policies for a continuous-review discrete material flow production/inventory system with fluctuating and correlated purchasing and sales prices, exponentially distributed raw material and demand inter-arrival times, and processing time. The sales and purchasing prices are driven by the random environmental changes that evolve according to a discrete state space continuous-time Markov process. We model the system as an infinitehorizon Markov decision process under the average reward criterion and prove that the optimal purchasing, production, and sales strategies are state-dependent threshold policies. We propose a linear programming formulation to compute the optimal threshold levels. We examine the effects of the sales price variation, purchasing price variation, correlation between sales and purchasing prices, customer arrival rate and limited inventory capacities on the system performance measures, through a range of numerical experiments. We also examine under which circumstances the use of the optimal policy notably improves the system profit compared to the use of the buy low and sell high naive policy. We show that using the optimal purchasing, production, and sales policies allow manufacturers to improve their profits when the purchasing and sales prices fluctuate.
\end{abstract}

Key words: Random environment, Price fluctuation, Markov decision process, Linear Programming

\section{Introduction}

In many industries, manufacturers of standard products purchase raw materials from commodity markets and sell their products at market prices that are determined by the external market

* It will be published IISE Transactions 


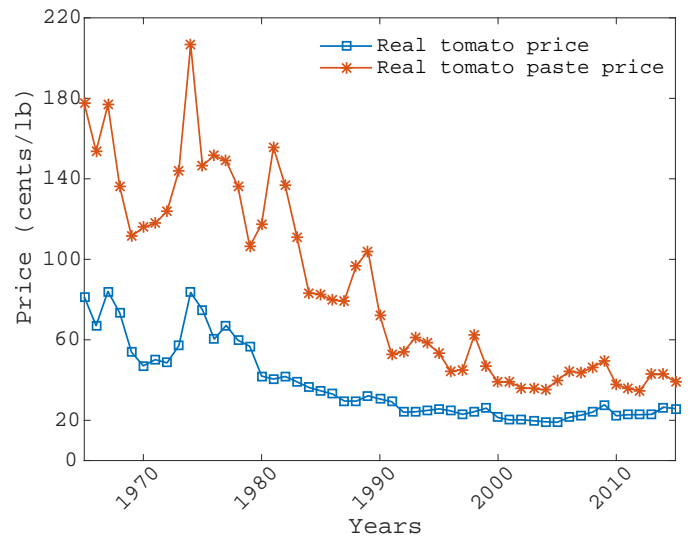

(a)

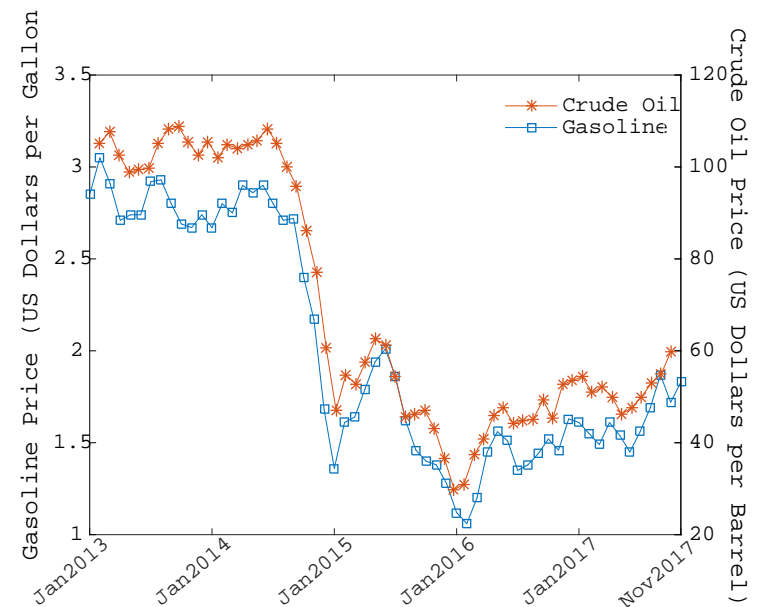

(b)

Figure 1 Figure 1(a) Yearly price for tomato paste and tomatoes in California U.S. Figure, from http://morningstarco.com/statdocs/2016\%20Exhibits\%20Brochure.pdf. Figure 1(b) Monthly rate of changes in the price of crude oil and in the price of gasoline, from https://data.worldbank.org/datacatalog/commodity-price-data.

conditions. As a result, their revenue and cost structures are directly affected by the volatility of purchasing and sales prices in the markets.

In particular, manufacturers whose products are related to the agricultural, mineral, oil, and energy sectors make transactions based on the commodity markets and must deal with the price fluctuations in both sales and purchasing operations. For instance, Figure1(a) shows how tomato and tomato paste prices change between the years 1965 and 2015. Figure1(b) also depicts the monthly rate of changes in the price of crude oil and in the price of gasoline between the years 2013 and 2017.

This dependence on market price fluctuations also occurs for industrial goods, such as D-RAM or white goods (Berling and Martínez-de Albéniz 2011). In addition, manufacturers that execute their purchasing, production, and sales operations in an international setting with different currencies have a similar issue because of the fluctuations in the spot rate of different currencies. Due to the high competition in the markets for standard products, manufacturers cannot individually exert an influence over the selling and purchasing prices. In this environment, reflecting the fluctuations in the purchasing prices directly on the sales price will not be accepted by the customers since the prices for the standard products are determined by the market conditions (Berling and Xie 2014). 
To cope with the volatility in the market prices, manufacturers can utilize an operational hedging strategy in terms of purchasing, production, and sales decisions. However, in the literature, the most of the production and inventory control models do not consider a need for the operational hedging strategies that handle the fluctuating market prices. Furthermore, to the best of our knowledge, none of the existing studies focus on the impacts of the correlation between sales and purchasing prices on a production/inventory system where the sales and purchasing prices together fluctuate in a random fashion.

Our aim in this article is developing an analytical model that can be used to determine the optimal purchasing, production, and sales strategies for a manufacturer with a finite capacity and explaining how the sales price variation, purchasing price variation, correlation between sales and purchasing prices, limited raw materials and finished goods inventories, and limited production capacity affect the performance of a production/inventory system. In this context, we focus on the following research questions:

- What are the optimal purchasing, production, and sales policies for a production/inventory system in which the sales and purchasing prices evolve according to the random environment changes?

- What are the impacts of sales price variation, purchasing price variation, correlation between sales and purchasing prices, limited raw materials and finished goods inventories, and limited production capacity on the system performance measures?

- Under which circumstances does the use of the optimal policy significantly improve the profit compared to the use of the buy low and sell high naive policy where the manufacturer buys only when the purchasing price is low and sells only when the sales price is high?

In order to answer these questions, we study a continuous-review discrete-material flow production/inventory system with raw materials and finished goods inventories and consider that the sales and purchasing prices are driven by a randomly fluctuating environment. In our study, the price fluctuations are represented by a discrete state space, continuous time Markov process. The 
demand and raw material arrival processes are characterized as Poisson processes and the production system with a limited capacity is modeled as a single server with exponential processing times. Under this problem setting, we model the system as an infinite-horizon Markov decision process under the average reward criterion.

To address the first research question, we employ the event-based dynamic programming approach. With this effort, we analytically prove that the optimal purchasing, production, and sales strategies are state-dependent threshold policies. We also propose a solution procedure based on a linear programming formulation to numerically identify the optimal threshold levels for purchasing, production and sales strategies.

We answer the second question by conducting a range of numerical experiments. As a result, we observe that the negative correlation between sales and purchasing prices yields a higher average reward for the manufacturer. The positive correlation between sales and purchasing prices lowers the average reward for the manufacturer while increases the average inventory and customer service levels. We notice that a higher variation in either the sales price or purchasing price yields a higher profit for the manufacturer. Additionally, we conclude that limited raw materials and finished goods inventories and limited production capacity have considerable effects on the system performance measures.

Finally, in order to address the last question, we first modify the linear programming formulation to model the operation of a producer that uses the buy low and sell high naive purchasing and sales policy but determines the production policy optimally. Then by using numerical experiments, we show that the high sales price variation, high purchasing price variation, and negative correlation yield a decrease in the difference between the profits achieved by using the optimal and naive policies.

We consider deriving the optimal purchasing, production, and sales policies for a production system operating with randomly fluctuating and correlated purchasing and sales prices and using the derived policies to show the impacts of sales price variation, purchasing price variation, correlation between sales and purchasing prices, limited raw materials and finished goods inventories, 
and limited production capacity on the system performance measures as the main contributions of this study.

The rest of this article is organized as follows: Section 2 reviews the related literature and contextualizes our contribution. Section 3 contains the model formulation and outlines the analytical results on the optimal purchasing, production, and sales policies. In Section 3, a linear programming formulation is also presented as a numerical tool to solve the model. Section 4 summarizes the results of the numerical analysis and the managerial insights derived from the analysis. Finally, the conclusions and future research directions are provided in Section 5.

\section{Literature Review}

In the literature, a number of studies typically examine either the impact of fluctuating sales price or of fluctuating purchasing price on the optimal purchasing, production or sales policies. In most of these studies, the optimal control models with the different time and state space representations are employed. Based on these representations, the current literature is categorized into several groups and each group is discussed in this review.

The discrete-time discrete-state space models in the literature primarily focus on inventory systems. As one of the early studies in this literature, Özekici and Parlar (1999) develop an infinitehorizon inventory model with unreliable suppliers, where the demand, supply, and cost parameters are affected by a randomly changing environment. In their model, the random changes in the environment states are modulated by a time-homogeneous Markov process. The authors show that an environment-dependent base-stock policy is optimal when the order cost is linear in order quantity. Additionally, an $(s, S)$ policy is found to be optimal when a fixed cost of ordering is in place. In the work of Özekici and Parlar (1999), the supplier is either fully available or unavailable based on the state of the environment. Erdem and Özekici (2002) extend this assumption by considering a case in which the supplier is always available, but its capacity is random and dependent on the state of the environment. Papachristos and Katsaros (2008) further the work of Erdem and Özekici (2002) by introducing a constant lead time and by considering the effect of stochastic ordering of the 
random variables that represent the supplier's capacity levels on the optimal policy parameters. Gavirneni (2004) considers an uncapacitated production/inventory system in which the purchasing cost fluctuates over the time due to the changes in the exchange rate. The purchasing cost fluctuations are modeled with a time-homogeneous Markov chain. The author proves the optimality of an order up to policy and proposes a simple myopic heuristic for finding the policy parameters. De Cuypere et al. (2013) study the optimal inventory control policy when the lead time of purchased parts follows a phase-type distribution and the purchasing price is a random variable that follows a Markovian process. Liu and Yang (2015) focus on a problem in which a make-to-stock firm periodically controls both the purchasing activity and sales price. The authors consider that the purchasing price randomly fluctuates according to a Markovian fashion and the demand is a random variable depending on the sales price. For the considered problem setting, they show that the optimal purchasing policy is a base-stock type.

In the literature, the continuous-time continuous-state space models are also employed to describe the fluctuations in sales and/or purchasing prices as Markov diffusion processes. Wu and Chen (2010) consider a problem setting where an individual firm controls production and two stages of inventory specifying the deterministic purchasing, production, and sales rates. The sales and purchasing prices are modeled as standard Brownian motion processes. Under this problem setting, the authors study the relation between the inventory and short-term price variation. They also characterize the rational expectations equilibrium for the market in which there are competitive production firms. Berling and Martínez-de Albéniz (2011) examine the optimality of a price-dependent base-stock policy when the demand is a Poisson process and the purchasing price evolves either as a geometric Brownian motion or an Ornstein-Uhlenbeck process. Berling and Xie (2014) propose a heuristic to handle the computational intractability issue raised while obtaining the parameters for the optimal policy suggested in the work of Berling and Martínez-de Albéniz (2011). In some of the studies that consider continuous-time continuous-state space framework, the fluctuations in purchasing or operating costs are defined as a deterministic function of time. Arnold 


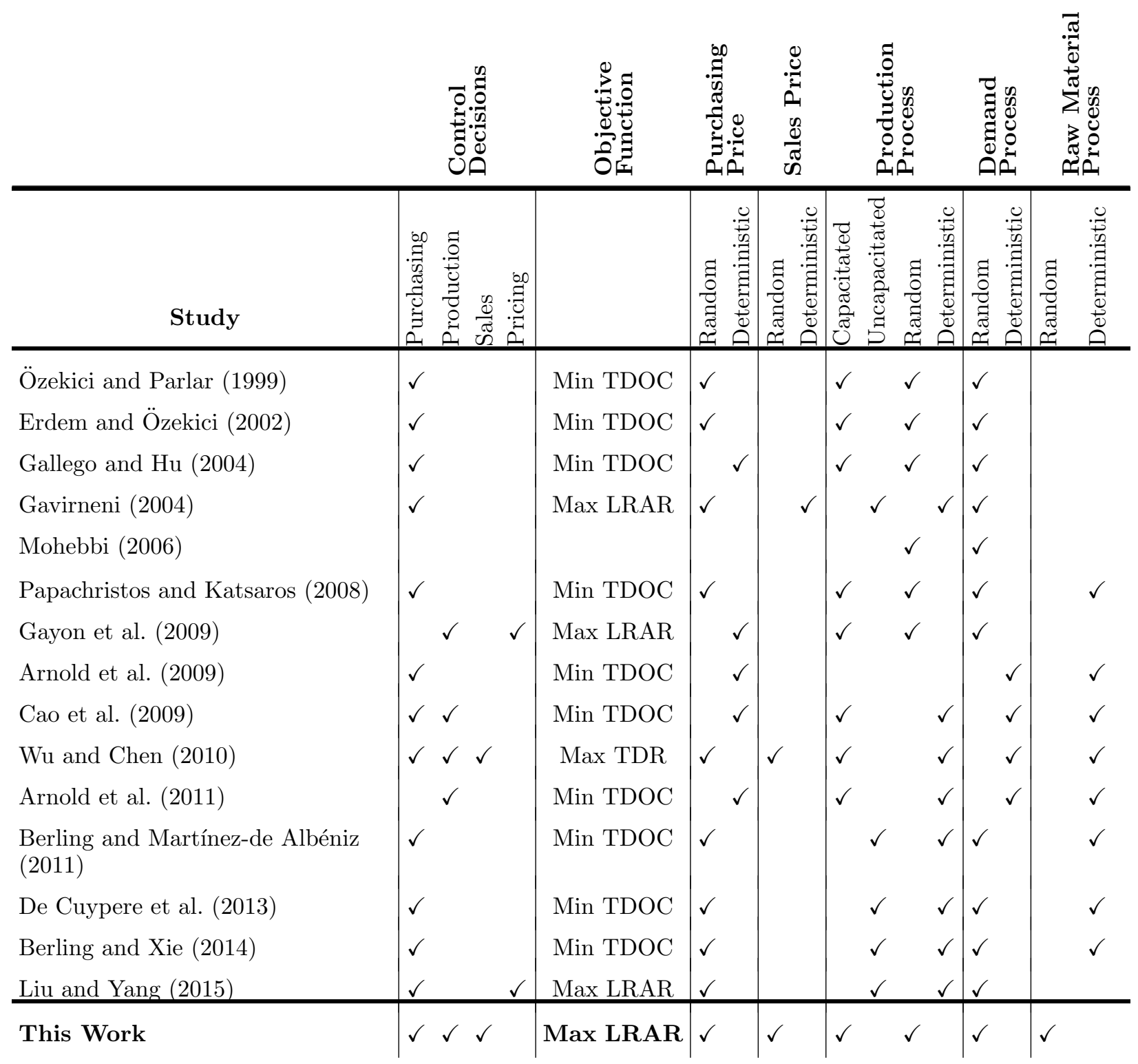

TDOC: Time Discounted Operating Cost, LRAR: Long-Run Average Reward, TDR: Time Discounted Reward

Table 1 A summary table for the reviewed studies

et al. (2009) study the optimal purchasing and inventory policies for a firm that operates in an environment where the ordering cost is zero and the purchasing price, demand, and inventory holding costs vary over time. Arnold et al. (2011) extend the analysis of Arnold et al. (2009) to a production model with finite production rate. Cao et al. (2009) consider a production/inventory system whose operations are subject to the time-dependent deterministic fluctuations in the demand, production cost, and purchasing cost over time. 
A number of studies have also employed the random environment framework to model the randomness in the supply and/or demand processes. In the context of this literature, Gallego and $\mathrm{Hu}(2004)$ study the optimal policies for production/inventory systems with finite capacity and Markov-Modulated demand and supply processes. Mohebbi (2006) focuses on a capacitated production/inventory system with compound Poisson demand, where both production and demand processes are subject to independently and randomly changing environmental conditions. The author derives the limiting distribution of the inventory level and provides a number of numerical results concerning the system performance measures. Gayon et al. (2009) consider a make-to-stock production/inventory system in which the customer demand is price-sensitive and its distribution depends on the state of the random environment. The authors study the effects of different pricing strategies on the system profit and derive the optimal replenishment policies for each considered pricing strategy.

Table 1 compares the studies discussed above and our work in different aspects. From this comparison, we note that the existing studies typically focus on either the downstream or upstream side of a production or inventory system. Since a production/inventory system with raw materials and finished goods inventories is considered in our work, we study downstream and upstream sides of the system together. In particular, most of the existing studies ignore the process of purchasing raw materials to facilitate the analysis. They typically assume that a raw material is always ready whenever it is required for the production. This assumption generally does not hold in practice because of the randomness in the delivery times in the supply process, the structure of the market, and the suppliers' limited capacities. Unlike most of the existing studies, we include the purchasing process in the system as a stochastic process.

With this effort, we believe that the model provides a better representation for a manufacturer operating in agricultural sector. In the agricultural sector, the raw material arrivals are random because of the harvest times, the geographic location of the suppliers, and weather conditions. In such a setting, the manufacturer generally decides on whether to buy the harvested raw materials 
or not. If the manufacturer decides not to buy she loses the current purchasing opportunity. When the raw material is required for the manufacturer she has to wait until the next harvest time. On the other hand, the manufacturer can reject the arriving customer even if the inventory on hand is positive because she may use the existing products for future sales at a higher price. This can be considered as a typical hoarding example in the agricultural sector. In addition, when the raw material arrivals rate is set to a sufficiently higher value than the other rates in the system the model allows us to analyze a setting where the raw material is almost always available for the manufacturer. Because, with this particular setting, the frequency of raw material arrivals increases thereby increasing the number of raw material purchase opportunities for the manufacturer. As a natural result of this fact, the system would resemble the one in which the manufacturer is able to buy a raw material whenever she needs it. Hence, the obtained results can be employed to derive inferences regarding the system where the raw materials are always available.

Table 1 also shows that the existing studies typically consider a random fluctuation in either sales or purchasing price. However, in our work, both sales and purchasing prices are subject to the random environment changes that evolve based on a continuous-time Markov chain. With this problem setting, we study the impacts of sales and purchasing price variations on the system performance measures. Additionally, unlike the existing studies, we address the effects of correlation between sales and purchasing prices on the system. In our work, the demand and raw material arrivals are considered as two independent Poisson processes. The production capacity is limited because a single server processes raw materials individually with exponential processing times.

To the best of our knowledge, this is the first attempt to derive the purchasing, production, and sales policies of a production/inventory system with correlated and fluctuating purchasing and sales prices, random demand and raw material arrivals and random processing time. With this effort, we are able to investigate the impacts of limited production capacity and traffic intensity on the system performance measures. The use of this modeling technique also allows us to model the system as an infinite-horizon Markov decision process under the average cost criterion. 




Figure 2 Illustration of the problem

\section{The Model and The Main Results}

In this section, we describe the problem setting and propose the dynamic programming model for the problem. Additionally, we derive the optimal purchasing, production, and sales policies for the manufacturer and the structural characteristics of these optimal control policies. A linear programming formulation is given to numerically obtain the parameters of the optimal purchasing, production, and sales policies.

\subsection{Problem definition}

We consider a discrete-material flow production/inventory system where a single manufacturer with a limited production capacity operates in a make-to-stock manner to fulfill the demand of a single item. Figure 2 illustrates the considered system.

When a unit of raw material released into the manufacturing facility, it is processed there and then placed into the finished goods inventory. The manufacturing facility is capable of processing one item at a time. For the processing of a unit of raw material, the manufacturer incurs the production cost of $w$. The processing time is exponentially distributed at the rate $\mu$.

Each customer arriving at the manufacturer requests only one unit of a finished good. Once a customer arrives to the facility, the manufacturer decides whether to satisfy the arriving customer from the finished goods inventory or reject it. That is, in order to keep the existing products for future sales at a higher price, the manufacturer does not have to satisfy an arriving demand even though there are some goods in the finished goods inventory. When the arriving demand is satisfied, the manufacturer receives the market sales price for this transaction. If the arriving demand is rejected then it is lost without a penalty cost. In the model, it is not necessary to consider a 
penalty cost for rejecting the arriving customer because the manufacturer takes this decision by considering a risk of the loss in the sales revenue. That is, the sales revenue loss that may arise due to rejecting the arriving customer can be considered as a penalty cost in the model. Additionally, a consideration of a penalty cost for rejecting the arriving customer would not change the optimal strategies and main findings in the model, however, it may decrease the value of expected profit function.

In order to analyze the purchasing policy in a discrete-material flow system, we model the system with raw materials arriving to the manufacturing facility individually with exponentially distributed inter-arrival times with the rate $\delta$ that is much higher compared to the other rates of change in the system. Once a unit of raw material arrives to the facility, the manufacturer decides whether to buy it or not. When the manufacturer decides to buy the arriving raw material, he pays the market purchasing price for this transaction and a unit of arriving raw material is added into the raw materials inventory.

The stock level of the raw materials inventory is $X_{1}(t)$ at time $t$ where $X_{1}(t) \in \mathbb{N}=\{0,1,2, \ldots\}$. The finished goods inventory level at time $t$ is denoted by $X_{2}(t)$ where $X_{2}(t) \in \mathbb{N}=\{0,1,2, \ldots\}$. A holding cost of keeping a unit of raw material in the raw materials inventory is $h_{1}$ per unit time whereas a holding cost of storing a unit of finished good in the finished goods inventory is $h_{2}$ per unit time. In the models where an upstream and a downstream inventory are used together, it is usually assumed that $h_{2}>h_{1}$ in order to reflect a higher value added for the products in the downstream inventory. In our model, since the purchasing and market prices fluctuate, we do not make this restricting assumption.

The manufacturer's operating environment is subject to random changes that directly affect the unit prices for the raw materials and finished goods. The environment state evolves according to a continuous-time homogeneous Markov chain with a transition rate matrix $Q$. The elements $q_{i j}$ in the transition matrix describe the rate departing from $i$ and arriving in state $j \neq i$. The state of the environment at time $t$ is represented by $I(t)$ where $I(t) \in \mathbb{E}=\{1,2, \ldots, L\}$. 
The manufacturer is a price-taker, that is, he cannot individually exert influence over the market prices of finished goods and raw materials. Therefore, the manufacturer uses the market price when he sells a finished good or procures a raw material. The market purchasing and sales prices depend on the state of the environment. Specifically, for the environment $I(t)=i \in \mathbb{E}$, the market prices for purchasing and sales transactions are $C(I(t))=c_{i}$ where $c_{i} \in \mathbb{C}=\left\{c_{1}, c_{2}, \ldots, c_{L}\right\}$ and $S(I(t))=s_{i}$ where $s_{i} \in \mathbb{S}=\left\{s_{1}, s_{2}, \ldots, s_{L}\right\}$, respectively. In the setting where the sales price is lower than the purchasing price, the manufacturer would prefer not to continue his operations since he cannot generate a profit. In order to avoid this trivial case, we consider that the lowest possible minimum sales price is greater than or equal to the highest possible purchasing price, i.e., $\min \left\{s_{1}, s_{2}, \ldots, s_{L}\right\} \geq$ $\max \left\{c_{1}, c_{2}, \ldots, c_{L}\right\}$. Specifically, it is profitable to purchase, produce, and sell in all environment states, but the profit margins are different.

The manufacturer is always able to observe the state of the environment, raw materials inventory level, and finished goods inventory level. Based on these observations, he seeks to find the optimal purchasing, production and sales policies that maximize his average reward per unit time over an infinite planning horizon.

\subsection{Optimal control model}

Since the system retains no memory as the time between each transition is exponentially distributed, the decision epochs in our model can be restricted to times when the state changes. Using these properties, we can formulate the problem as a Markov decision process (MDP) and restrict our attention only to the class of stationary Markovian policies (Puterman 2014). For the class of stationary Markovian policies, the actions at each decision epoch depend solely on the current state. The current state of the system is therefore described as independent of time $t$ and denoted by the variable $(i, \underline{\mathbf{x}})$. In the state variable, $i$ is the state of the environment and it belongs to the set of $\mathbb{E}$. In addition, $\underline{\mathbf{x}}$ represents the stock levels of the raw materials and finished goods inventories and it belongs to set of $\mathbb{F}=\left\{\left(x_{1}, x_{2}\right) \mid x_{k} \in \mathbb{N}\right.$ where $\left.k \in\{1,2\}\right\}$. Hence, the state variable, $(i, \underline{\mathbf{x}})$, belongs to the state space $\mathbb{E} \times \mathbb{N}^{2}$. 
For any given state, the manufacturer may choose either to purchase a raw material or not to purchase. These two actions are denoted by 1 and 0 , respectively, and the action taken by $u_{b}$. If an action $u_{b}=1$ is taken when the environment state is $i$, a cost of $c_{i}$ is incurred. He might also choose either to produce or not to produce. These two actions are represented by 1 and 0 , respectively, and the action taken by $u_{p}$. When an action $u_{p}=1$ is chosen, he pays a unit production cost $w$. When a demand arrives, it may either be immediately satisfied or rejected. These two actions are denoted by 1 and 0 , respectively, and the action taken by $u_{r}$. If an action $u_{r}=1$ is taken when the environment state is $i$, a revenue of $s_{i}$ is accrued.

For each state $(i, \underline{\mathbf{x}})$, a control policy $\ell$ specifies the action $u^{\ell}(i, \underline{\mathbf{x}})=\left(u_{b}, u_{p}, u_{r}\right)$. Given a policy $\ell$ and an initial state $(i, \underline{\mathbf{x}})$, the manufacturer's average reward per unit time over an infinite planning horizon is defined as

$$
v^{\ell}(i, \underline{\mathbf{x}})=\lim _{T \rightarrow \infty} \frac{1}{T} \mathrm{E}_{(i, \underline{\mathbf{x}})}^{\ell}\left[\int_{0}^{T} S(I(t)) d N_{R}(t)-\int_{0}^{T} w d N_{P}(t)-\int_{0}^{T} H\left(X_{1}(t), X_{2}(t)\right) d t-\int_{0}^{T} C(I(t)) d N_{B}(t)\right] .
$$

In the above equation, $H\left(X_{1}, X_{2}\right)=h_{1} X_{1}(t)+h_{2} X_{2}(t)$ is the total holding cost function, $N_{R}(t)$ is the number of customers satisfied up to $t, N_{P}(t)$ is the number of products produced up to $t$, and $N_{B}(t)$ is the number of raw materials purchased up to $t$. Additionally, $\mathrm{E}_{(i, \underline{\mathbf{x}})}^{\ell}$ denotes the expectation operation given an initial state $(i, \underline{\mathbf{x}})$ and a policy $\ell$. The objective is to identify a control policy $\ell^{*}$ that satisfies $v^{*}(i, \underline{\mathbf{x}})=\sup _{\ell} v^{\ell}(i, \underline{\mathbf{x}})$ for all states.

Following Lippman (1975), we work with a uniformized version of the original problem in which the transition rate in each state under any action is $\Lambda=\delta+\lambda+\mu+\sum_{i} \sum_{j \neq i} q_{i j}$ so that the transition times between decision epochs form a sequence of i.i.d. exponential random variables, each with a mean of $1 / \Lambda$. The introduction of the uniform transition rate allows us to build a discrete-time equivalent of the original system, simplifying the analysis considerably. To further simplify the analysis, we also rescale the time by letting $\Lambda=1$. As a result of these efforts, we obtain the following optimality equation:

$$
v(i, \underline{\mathbf{x}})+g^{*}=\delta T_{b} v(i, \underline{\mathbf{x}})+\mu T_{p} v(i, \underline{\mathbf{x}})+\lambda T_{r} v(i, \underline{\mathbf{x}})+T_{e} v(i, \underline{\mathbf{x}})-h \underline{\mathbf{x}} .
$$


In the above equation, $h=\left(h_{1}, h_{2}\right)^{T}, g^{*}$ is the optimal average reward, and $v(i, \underline{\mathbf{x}})$ denotes the differential reward between the current reward and the optimal average reward. The operators $T_{b} f(i, \underline{\mathbf{x}}), T_{p} f(i, \underline{\mathbf{x}}), T_{r} f(i, \underline{\mathbf{x}})$, and $T_{e} f(i, \underline{\mathbf{x}})$ for any real-valued function $f(i, \underline{\mathbf{x}})$ are defined as follows:

$$
\begin{aligned}
& T_{b} f(i, \underline{\mathbf{x}})=\max \left\{f\left(i, \underline{\mathbf{x}}+e_{1}\right)-c_{i}, f(i, \underline{\mathbf{x}})\right\}, \\
& T_{p} f(i, \underline{\mathbf{x}})= \begin{cases}\max \left\{f\left(i, \underline{\mathbf{x}}-e_{1}+e_{2}\right)-w, f(i, \underline{\mathbf{x}})\right\}, & x_{1}>0, \\
f(i, \underline{\mathbf{x}}), & x_{1}=0,\end{cases} \\
& T_{r} f(i, \underline{\mathbf{x}})= \begin{cases}\max \left\{f\left(i, \underline{\mathbf{x}}-e_{2}\right)+s_{i}, f(i, \underline{\mathbf{x}})\right\}, & x_{2}>0, \\
f(i, \underline{\mathbf{x}}), & x_{2}=0,\end{cases} \\
& T_{e} f(i, \underline{\mathbf{x}})=\sum_{j \in \mathbb{E} \backslash\{i\}} q_{j i} f(j, \underline{\mathbf{x}}),
\end{aligned}
$$

where $e_{1}$ is $(1,0)$ and $e_{2}$ is $(0,1)$.

The operators $T_{b} f(i, \underline{\mathbf{x}}), T_{p} f(i, \underline{\mathbf{x}})$, and $T_{r} f(i, \underline{\mathbf{x}})$ respectively correspond to the purchasing, production, and sales decisions whereas $T_{e} f(i, \underline{\mathbf{x}})$ specifies the environmental changes. The state trajectory of the system corresponding to a unichain policy is eventually confined to the recurrent class of states. Because, the state space of the environment is finite and the manufacturer controls the purchasing, production and sales events. Since the system has this unique property, the average reward corresponding to all initial states as well as the differential rewards of the recurrent states are independent of the rewards obtained from the transient states (Bertsekas 1995). This result assures us that the Bellman's equation $v^{*}+g^{*}=T v^{*}$ holds and there exists a unique $g^{*}$.

\subsection{Characterization of the optimal purchasing, production, and sales policies}

To define the optimality equation given in the previous section, we have employed the event-based dynamic programming approach that is originally proposed by Koole (1998). In the optimality equation, each certain event in the system is represented by a specific event operator. Representing each event with a specific event operator allows us to analyze the propagation of value function 
properties through the propagation properties of the event operators. Specifically, if the event operators have some structural properties under the given assumptions, then the value function of the system constructed by using these operators also has the same structural properties.

By using the structure of the event-based dynamic programming approach and the results obtained in the works of Altman et al. (2000) and Koole (2004), we establish that the optimal purchasing, production, and sales policies for the manufacturer are state-dependent threshold policies. In Theorem 1, 3, and 5, we propose the details related to the optimal purchasing, production, and sales policies, respectively. We also establish the monotonicity properties of the optimal purchasing, production, and sales policies in Theorem 2, 4, and 6. In the Appendix A, we present a detailed discussion on the proofs of the theorems.

THEOREM 1. The optimal purchasing policy for the manufacturer is a state-dependent threshold policy. That is, for any given state $\left(i^{\prime},\left(x_{1}^{\prime}, x_{2}^{\prime}\right)\right)$, there exists a corresponding threshold level, $Z_{b}^{*}\left(i^{\prime}, x_{2}^{\prime}\right)$. It is optimal to buy if $x_{1}^{\prime}<Z_{b}^{*}\left(i^{\prime}, x_{2}^{\prime}\right)$ and to do nothing otherwise, i.e.,

$$
u_{b}^{*}\left(i^{\prime},\left(x_{1}^{\prime}, x_{2}^{\prime}\right)\right)= \begin{cases}1, & \text { if } x_{1}^{\prime}<Z_{b}^{*}\left(i^{\prime}, x_{2}^{\prime}\right), \\ 0, & \text { if } x_{1}^{\prime} \geq Z_{b}^{*}\left(i^{\prime}, x_{2}^{\prime}\right) .\end{cases}
$$

To clarify the theorems, we use a problem instance analyzed optimally by using the approach given in Section 3.4 and characterize its optimal purchasing, production, and sales policies. In Figure 3(a), we give the optimal purchasing policy for one of the environment states considered in the example denoted with the environment state $i^{\prime \prime}$. Let us consider the state where there are 2 units in the raw material and 2 units in the finished goods inventories, i.e., the state $\left(i^{\prime \prime},(2,2)\right)$. Theorem 1 implies that the purchasing threshold level for the given state is one of the points at $\left(i^{\prime \prime},\left(x_{1}, 2\right)\right)$ where $x_{1} \in \mathbb{N}$. Figure $3\left(\right.$ a) shows that the corresponding threshold level is $Z_{b}^{*}\left(i^{\prime \prime}, 2\right)=5$. Theorem 1 also implies that for all the points at $\left(i^{\prime \prime},\left(x_{1}, 2\right)\right)$ where $x_{1} \in \mathbb{N}$, the purchasing threshold level is $Z_{b}^{*}\left(i^{\prime \prime}, 2\right)=5$. That is, for any given point $\left(i^{\prime \prime},\left(x_{1}, 2\right)\right)$ where $x_{1} \in \mathbb{N}$, it is optimal to purchase if $x_{1}<5$ and not to purchase if $x_{1} \geq 5$. 


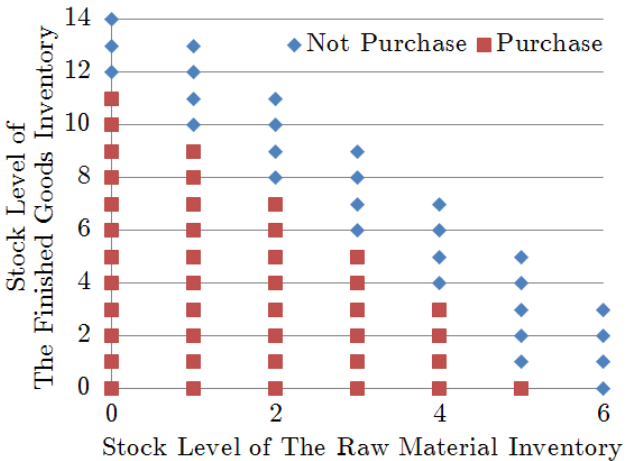

(a)

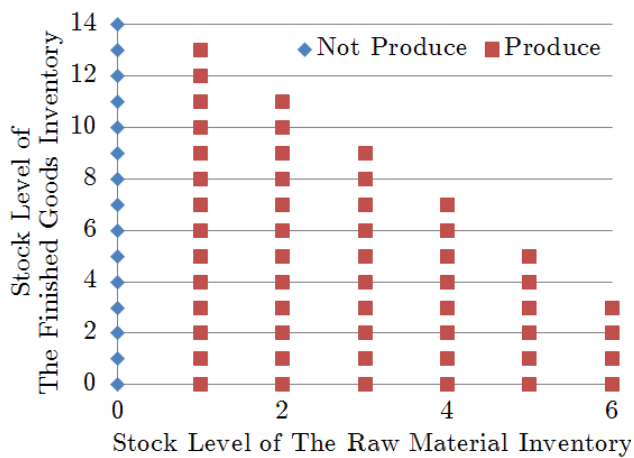

(b)

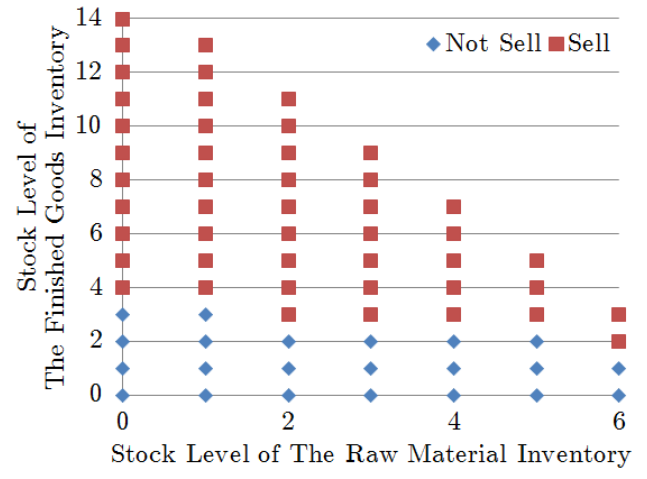

(c)

Figure 3 An illustration of the optimal 3(a) purchasing, 3(b) production, and 3(c) sales strategies derived for a particular environment state

With the introduced problem instance, we observe that for any fixed $i$ and $x_{2}$, there exists a corresponding threshold level for purchasing decision and it is not optimal to buy a raw material when the raw materials inventory level for a given state is above a certain threshold level. To discuss this implication in more detail, let us modify the manufacturer's maximization problem into an equivalent minimization problem. In the modified version of the problem, the operator related to purchasing decision is defined as $T_{b}^{\prime} v^{\prime}(i, \underline{\mathbf{x}})=\min \left\{v^{\prime}\left(i, \underline{\mathbf{x}}+e_{1}\right)+c_{i}, v^{\prime}(i, \underline{\mathbf{x}})\right\}$ where $v^{\prime}(i, \underline{\mathbf{x}})=-v(i, \underline{\mathbf{x}})$. Also, let us define an operator $\nabla v^{\prime}(i, \underline{\mathbf{x}})=v^{\prime}\left(i, \underline{\mathbf{x}}+e_{1}\right)-v^{\prime}(i, \underline{\mathbf{x}})$. Intuitively, for the modified problem, $\nabla v^{\prime}(i, \underline{\mathbf{x}})$ represents the benefit of having an extra raw material in the raw materials inventory when the environment state is $i . T_{b}^{\prime} v^{\prime}(i, \underline{\mathbf{x}})$ implies that it is optimal not to buy a raw material in state $(i, \underline{\mathbf{x}})$ if $\nabla v^{\prime}(i, \underline{\mathbf{x}})>-c_{i}$. With the results obtained in the works of Koole (2004) and Altman et al. (2000), we also prove that the benefit of an extra item in the raw materials inventory is non-decreasing in $x_{1}$ for each environment state $i$ when $v^{\prime}(i, \underline{\mathbf{x}})$ is multimodular. Whenever this 
benefit exceeds above a certain value, more specifically above the purchasing $\operatorname{cost}-c_{i}$, it will remain above $-c_{i}$. This fact guarantees the existence of a threshold level for the purchasing decision. A similar discussion can also be adapted to Theorem 3 and Theorem 5 .

THEOREM 2. The optimal purchasing policy for the manufacturer is non-increasing in $x_{1}$ and $x_{2}$. That is, $Z_{b}^{*}\left(i, x_{2}\right)$ that defines the purchasing threshold level for any given state $(i, \underline{\mathbf{x}})$ is nonincreasing in $x_{2}$.

Let us consider the state where there are 2 units in the raw material and 5 units in the finished goods inventories, i.e., the state $\left(i^{\prime \prime},(2,5)\right)$. Theorem 2 implies that for any given state $\left(i^{\prime \prime},\left(2, x_{2}^{\prime \prime}\right)\right)$ where $x_{2}^{\prime \prime}>5$ the inequality $u_{b}^{*}\left(i^{\prime \prime},(2,5)\right) \geq u_{b}^{*}\left(i^{\prime \prime},\left(2, x_{2}^{\prime \prime}\right)\right)$ holds. In Figure $3(\mathrm{a})$, we observe that the optimal purchasing policy in the state $\left(i^{\prime \prime},(2,5)\right)$ satisfies the given inequality, i.e., $u_{b}^{*}\left(i^{\prime \prime},(2,5)\right)=$ $1 \geq u_{b}^{*}\left(i^{\prime \prime},(2,6)\right)=1 \geq u_{b}^{*}\left(i^{\prime \prime},(2,7)\right)=1 \geq u_{b}^{*}\left(i^{\prime \prime},(2,8)\right)=0$. Let us also consider that $Z_{b}^{*}\left(i^{\prime \prime}, 5\right)$ and $Z_{b}^{\prime *}\left(i^{\prime \prime}, x_{2}^{\prime \prime}\right)$ are the purchasing threshold levels for the state $\left(i^{\prime \prime},(2,5)\right)$ and any one of the states that satisfies $\left(i^{\prime \prime},\left(2, x_{2}^{\prime \prime}\right)\right)$ where $x_{2}^{\prime \prime}>5$. Theorem 2 implies that $Z_{b}^{*}\left(i^{\prime \prime}, 5\right) \geq Z_{b}^{\prime *}\left(i^{\prime \prime}, x_{2}^{\prime \prime}\right)$ holds due to the implication on the optimal purchasing policy and Equation (3). For instance, in Figure $3(\mathrm{a})$, the purchasing threshold levels for the states $\left(i^{\prime \prime},(2,5)\right)$ and $\left(i^{\prime \prime},(2,6)\right)$ are $Z_{b}^{*}\left(i^{\prime \prime}, 5\right)=4$ and $Z_{b}^{\prime *}\left(i^{\prime \prime}, 6\right)=3$.

THEOREM 3. The optimal production policy for the manufacturer is a state-dependent threshold policy. That is, for any given state $\left(i^{\prime},\left(x_{1}^{\prime}, x_{2}^{\prime}\right)\right)$ where $x_{1}^{\prime}+x_{2}^{\prime}=k$, there exists a corresponding threshold level, $Z_{p}^{*}\left(i^{\prime},\left(x_{1}^{\prime}, x_{2}^{\prime}\right)\right)$. It is optimal to produce if $x_{1}^{\prime}>Z_{p}^{*}\left(i^{\prime},\left(x_{1}^{\prime}, x_{2}^{\prime}\right)\right)$ and $x_{2}^{\prime}<k-$ $Z_{p}^{*}\left(i^{\prime},\left(x_{1}^{\prime}, x_{2}^{\prime}\right)\right)$ and to stay idle otherwise, i.e.,

$$
u_{p}^{*}\left(i^{\prime},\left(x_{1}^{\prime}, x_{2}^{\prime}\right)\right)= \begin{cases}1, & \text { if } x_{1}^{\prime}>Z_{p}^{*}\left(i^{\prime},\left(x_{1}^{\prime}, x_{2}^{\prime}\right)\right) \text { and } x_{2}^{\prime}<k-Z_{p}^{*}\left(i^{\prime},\left(x_{1}^{\prime}, x_{2}^{\prime}\right)\right), \\ 0, & \text { if } x_{1}^{\prime} \leq Z_{p}^{*}\left(i^{\prime},\left(x_{1}^{\prime}, x_{2}^{\prime}\right)\right) \text { and } x_{2}^{\prime} \geq k-Z_{p}^{*}\left(i^{\prime},\left(x_{1}^{\prime}, x_{2}^{\prime}\right)\right) .\end{cases}
$$

In Figure 3(b), we give the optimal production policy for one of the environment states, $i^{\prime \prime}$, considered in the example. Let us consider the state where there are again 2 parts in the raw materials inventory and 2 parts in the finished goods inventory, i.e., the state $\left(i^{\prime \prime},(2,2)\right)$. Theorem 3 implies 
that the production threshold level for the given state is one of the points at $\left(i^{\prime \prime},\left(x_{1}, 4-x_{1}\right)\right)$ where $x_{1} \in \mathbb{N}$. Specifically, we can find the corresponding threshold value in Figure 3(b) by searching the line passing through the point $\left(i^{\prime \prime},(2,2)\right)$ with a slope of -1 . Figure $3(\mathrm{~b})$ shows that the production threshold level for the given state is $Z_{p}^{*}\left(i^{\prime \prime},(2,2)\right)=0$. Theorem 3 also implies that for all the points at $\left(i^{\prime \prime},\left(x_{1}, x_{2}\right)\right)$ where $x_{1}+x_{2}=4$, the production threshold level is $Z_{p}^{*}\left(i^{\prime \prime},\left(x_{1}, x_{2}\right)\right)=0$. That is, for any given $\left(i^{\prime \prime}, x_{1}, x_{2}\right)$ where $x_{1}+x_{2}=4$, it is optimal to produce if $x_{1}>0$ and $x_{2}<4$, and not to produce if $x_{1} \leq 0$ and $x_{2} \geq 4$.

THEOREM 4. The optimal production policy for the manufacturer is non-decreasing in $x_{1}$ and non-increasing in $x_{2}$. That is, $Z_{p}^{*}\left(i,\left(x_{1}, x_{2}\right)\right)$ that defines the production threshold level for the given state $\left(i,\left(x_{1}, x_{2}\right)\right)$ is non-increasing in $x_{2}$.

Let us consider the state where there are only 2 parts in the finished goods inventory, i.e., the state $\left(i^{\prime \prime}, 0,2\right)$. Theorem 4 implies that for any given state $\left(i^{\prime \prime},\left(0, x_{2}^{\prime \prime}\right)\right)$ where $x_{2}^{\prime \prime}>2$ the inequality $u_{p}^{*}\left(i^{\prime \prime},(0,2)\right) \geq u_{p}^{*}\left(i^{\prime \prime},\left(0, x_{2}^{\prime \prime}\right)\right)$ holds. From Theorem 4 , we also know that for any given state $\left(i^{\prime \prime},\left(x_{1}^{\prime \prime}, 2\right)\right)$ where $x_{1}^{\prime \prime}>0$ the inequality $u_{p}^{*}\left(i^{\prime \prime},(0,2)\right) \leq u_{p}^{*}\left(i^{\prime \prime},\left(x_{1}^{\prime \prime}, 2\right)\right)$ holds. In Figure 3(a), we observe that the optimal production policy obtained in the state $\left(i^{\prime \prime},(0,2)\right)$ satisfies the given both inequalities, i.e., $u_{p}^{*}\left(i^{\prime \prime},(0,2)\right)=0 \leq u_{p}^{*}\left(i^{\prime \prime},(1,2)\right)=1 \leq u_{p}^{*}\left(i^{\prime \prime},(2,2)\right)=1$ and $u_{p}^{*}\left(i^{\prime \prime},(0,2)\right)=$ $0 \geq u_{p}^{*}\left(i^{\prime \prime},(0,3)\right)=0 \geq u_{p}\left(i^{\prime \prime},(0,4)\right)=0$. Let us also consider that $Z_{p}^{*}\left(i^{\prime \prime},(0,2)\right)$ and $Z_{p}^{\prime *}\left(i^{\prime \prime},\left(0, x_{2}^{\prime \prime}\right)\right)$ are the production threshold levels for the state $\left(i^{\prime \prime},(0,2)\right)$ and any one of the states that satisfies $\left(i^{\prime \prime},\left(0, x_{2}^{\prime \prime}\right)\right)$ where $x_{2}^{\prime \prime}>2$. Theorem 4 implies that $Z_{p}^{*}\left(i^{\prime \prime},(0,2)\right) \leq Z_{p}^{\prime *}\left(i^{\prime \prime},\left(0, x_{2}^{\prime \prime}\right)\right)$ holds due to the implication on the optimal production policy and Equation (4). For instance, in Figure 3(b), the production threshold levels for the states $\left(i^{\prime},(0,2)\right)$ and $\left(i^{\prime},(0,3)\right)$ are $Z_{p}^{*}\left(i^{\prime \prime},(0,2)\right)=0$ and $Z_{p}^{\prime *}\left(i^{\prime \prime},(0,3)\right)=0$.

THEOREM 5. The optimal sales policy for the manufacturer is a state-dependent threshold policy. That is, for any given state $\left(i^{\prime},\left(x_{1}^{\prime}, x_{2}^{\prime}\right)\right)$, there exists a corresponding threshold level, $Z_{r}^{*}\left(i^{\prime}, x_{1}^{\prime}\right)$. It is optimal to sell if $x_{2}^{\prime}>Z_{r}^{*}\left(i^{\prime}, x_{1}^{\prime}\right)$, and to do nothing otherwise, i.e.,

$$
u_{r}^{*}\left(i^{\prime},\left(x_{1}^{\prime}, x_{2}^{\prime}\right)\right)= \begin{cases}1, & \text { if } x_{2}^{\prime}>Z_{r}^{*}\left(i^{\prime}, x_{1}^{\prime}\right), \\ 0, & \text { if } x_{2}^{\prime} \leq Z_{r}^{*}\left(i^{\prime}, x_{1}^{\prime}\right) .\end{cases}
$$


In Figure 3(c), we give the optimal sales policy for one of the environment states considered in the example, say, the environment state $i^{\prime \prime}$. Let us consider that $\left(i^{\prime \prime},(1,2)\right)$ is the given state. Theorem 5 implies that the sales threshold level for the given state is one of the points at $\left(i^{\prime \prime},\left(1, x_{2}\right)\right)$ where $x_{2} \in \mathbb{N}$. Figure $3(\mathrm{c})$ shows that the corresponding threshold level is $Z_{r}^{*}\left(i^{\prime \prime}, 1\right)=3$. Theorem 5 also implies that for all the points at $\left(i^{\prime \prime},\left(1, x_{2}\right)\right)$ where $x_{2} \in \mathbb{N}$, the sales threshold level is $Z_{r}^{*}\left(i^{\prime}, 1\right)=3$. That is, for any given point $\left(i^{\prime \prime},\left(1, x_{2}\right)\right)$ where $x_{2} \in \mathbb{N}$, it is optimal to sell if $x_{2}>3$ and not to sell if $x_{2} \leq 3$.

THEOREM 6. The optimal sales policy for the manufacturer is non-decreasing in $x_{1}$ and $x_{2}$. That is, $Z_{r}^{*}\left(i, x_{1}\right)$ that defines the sales threshold level for the given state $\left(i,\left(x_{1}, x_{2}\right)\right)$ is non-increasing in $x_{1}$.

Let us consider the state where there are only 3 units in the finished goods inventories, i.e., the state $\left(i^{\prime \prime},(0,3)\right)$. Theorem 6 implies that for any given state $\left(i^{\prime \prime},\left(x_{1}^{\prime \prime}, 3\right)\right)$ where $x_{1}^{\prime \prime}>0$ the inequality $u_{r}^{*}\left(i^{\prime \prime},(0,3)\right) \leq u_{r}^{*}\left(i^{\prime \prime},\left(x_{1}^{\prime \prime}, 3\right)\right)$ holds. In Figure $3(\mathrm{c})$, we observe that the optimal sales policy obtained in the state $\left(i^{\prime \prime},(0,3)\right)$ satisfies the given inequality, i.e., $u_{r}^{*}\left(i^{\prime \prime},(0,3)\right)=0 \leq u_{r}^{*}\left(i^{\prime \prime},(1,3)\right)=0 \leq$ $u_{r}^{*}\left(i^{\prime \prime},(2,3)\right)=1$. Let us also consider that $Z_{r}^{*}\left(i^{\prime \prime}, 0\right)$ and $Z_{r}^{* *}\left(i^{\prime \prime}, x_{1}^{\prime \prime}\right)$ are the sales threshold levels for the state $\left(i^{\prime \prime},(0,3)\right)$ and any one of the states that satisfies $\left(i^{\prime \prime},\left(x_{1}^{\prime \prime}, 3\right)\right)$ where $x_{1}^{\prime \prime}>0$. Theorem 6 implies that $Z_{r}^{*}\left(i^{\prime \prime}, 0\right) \geq Z_{r}^{\prime *}\left(i^{\prime \prime}, x_{1}^{\prime \prime}\right)$ holds due to the implication on the optimal sales policy and Equation (5). For instance, in Figure 3(a), the sales threshold levels for the states $\left(i^{\prime \prime},(0,3)\right)$ and $\left(i^{\prime \prime},(2,3)\right)$ are $Z_{r}^{*}\left(i^{\prime \prime}, 0\right)=3$ and $Z_{b}^{\prime *}\left(i^{\prime \prime}, 2\right)=2$.

\subsection{Linear programming formulation for the optimal control model}

We use a linear programming formulation to validate the optimal purchasing, production, and sales policies derived in the previous section, as well as to numerically compute the policy parameters. The main advantage of this approach is that it derives the steady state probabilities for the system without an extra effort (Bertsekas 1995). This fact allows us to readily examine the effects of system parameters on the performance measures. All of the sets and indices used in the linear programming formulation are listed in below. 


\section{$\underline{\text { Sets \& Indices }}$}

$u_{b}$ : Purchasing decision, $u_{b} \in \mathbb{B}=\{0,1\} \quad x_{1}$ : Raw materials inventory level, $x_{1} \in \mathbb{X}_{1}=\left\{0,1, \ldots, M_{1}\right\}$

$u_{p}$ : Production decision, $u_{p} \in \mathbb{P}=\{0,1\} \quad x_{2}$ : Finished goods inventory level, $x_{2} \in \mathbb{X}_{2}=\left\{0,1, \ldots, M_{2}\right\}$

$u_{r}$ : Sales decision, $u_{r} \in \mathbb{R}=\{0,1\} \quad i$ : Environment state, $i \in \mathbb{E}=\{1,2, \ldots, L\}$

$\underline{\mathbf{x}}$ : Inventory levels state, $\underline{\mathbf{x}} \in \mathbb{F}^{\prime}=\left\{\left(x_{1}, x_{2}\right) \mid x_{k} \in \mathbb{X}_{k}\right.$ where $\left.k \in\{1,2\}\right\}$

Table 2 Sets and indices used in the linear programming formulation

As a computational requirement of the linear programming approach, we should restrict the state spaces for the stock levels of the raw materials and finished goods inventories (Nadar et al. 2014). Therefore, we describe two new parameters $M_{1}$ and $M_{2}$ that are inventory truncation levels for the raw materials and finished goods inventories, respectively. These two truncation levels are chosen sufficiently high so that the globally optimal reward does not change with a further increase in one of them. With these efforts, the variables that denote the stock levels of the raw materials and finished goods inventories belong the sets $x_{1} \in \mathbb{X}_{1}=\left\{0,1,2, \ldots, M_{1}\right\}$ and $x_{2} \in \mathbb{X}_{2}=\left\{0,1,2, \ldots, M_{2}\right\}$, respectively. As a result, $\underline{\mathrm{x}}$ that represents the inventory levels in the state variable belongs to the set $\mathbb{F}^{\prime}$, i.e., $\underline{\mathbf{x}} \in \mathbb{F}^{\prime}=\left\{\left(x_{1}, x_{2}\right) \mid x_{k} \in \mathbb{X}_{k}\right.$ where $\left.k \in\{1,2\}\right\}$.

The decision variable for the LP formulation is denoted by $Y_{i, \underline{\mathbf{x}}, u_{b}, u_{p}, u_{r}}$. The variable gives us the long-run fraction of the time that the system is in state $(i, \underline{\mathbf{x}})$ when the actions $u_{b}, u_{p}$, and $u_{r}$ are taken. With this decision variable and the sets and indices introduced in the above, the LP formulation for the problem is given as follows:

$$
\max \phi=\sum_{i \in \mathbb{E}} \sum_{\underline{\mathbf{x}} \in \mathbb{F}^{\prime}} \sum_{u_{b} \in \mathbb{B}} \sum_{u_{p} \in \mathbb{P}} \sum_{u_{r} \in \mathbb{R}} Y_{i, \underline{\mathbf{x}}, u_{b}, u_{p}, u_{r}}\left(\lambda u_{r} s_{i}-\delta u_{b} c_{i}-\mu u_{p} w-x_{1} h_{1}-x_{2} h_{2}\right),
$$

subject to

$$
\begin{aligned}
\sum_{u_{b} \in \mathbb{B}} \sum_{u_{p} \in \mathbb{P}} \sum_{u_{r} \in \mathbb{R}}\left(Y_{i, \underline{\mathbf{x}}, u_{b}, u_{p}, u_{r}}-u_{b} \delta Y_{i, \underline{\mathbf{x}}-e_{1}, u_{b}, u_{p}, u_{r}}-u_{p} \mu Y_{i, \underline{\mathbf{x}}+e_{1}-e_{2}, u_{b}, u_{p}, u_{r}}-u_{r} \lambda Y_{i, \underline{\mathbf{x}}+e_{2}, u_{b}, u_{p}, u_{r}}\right. \\
\left.\quad-\sum_{j \in \mathbb{E} \backslash\{i\}} q_{j i} Y_{j, \underline{\mathbf{x}}, u_{b}, u_{p}, u_{r}}-\left(1-u_{b} \delta-u_{p} \mu-u_{r} \lambda-\sum_{j \in \mathbb{E} \backslash\{i\}} q_{i j}\right) Y_{i, \underline{\mathbf{x}}, u_{b}, u_{p}, u_{r}}\right)=0, \forall i \in \mathbb{E}, \forall \underline{\mathbf{x}} \in \mathbb{F}^{\prime}, \\
\sum_{i \in \mathbb{E}} \sum_{\underline{\mathbf{x}} \in \mathbb{F}^{\prime}} \sum_{u_{b} \in \mathbb{B}} \sum_{u_{p} \in \mathbb{P}} \sum_{u_{r} \in \mathbb{R}} Y_{i, \underline{\mathbf{x}}, u_{b}, u_{p}, u_{r}}=1,
\end{aligned}
$$




$$
\begin{aligned}
& Y_{i, \underline{\mathbf{x}}, u_{b}, u_{p}, u_{r}}=0, \forall i \in \mathbb{E}, \forall \underline{\mathbf{x}} \in\left\{\left(x_{1}, x_{2}\right) \mid x_{1}=0, x_{2} \in \mathbb{X}_{2}\right\}, \forall u_{b} \in \mathbb{B}, u_{p} \in\{1\}, \forall u_{r} \in \mathbb{R}, \\
& Y_{i, \underline{\mathbf{x}}, u_{b}, u_{p}, u_{r}}=0, \forall i \in \mathbb{E}, \forall \underline{\mathbf{x}} \in\left\{\left(x_{1}, x_{2}\right) \mid x_{1} \in \mathbb{X}_{1}, x_{2}=0\right\}, \forall u_{b} \in \mathbb{B}, \forall u_{p} \in \mathbb{P}, u_{r} \in\{1\}, \\
& Y_{i, \underline{\mathbf{x}}, u_{b}, u_{p}, u_{r}}=0, \forall i \in \mathbb{E}, \forall \underline{\mathbf{x}} \in\left\{\left(x_{1}, x_{2}\right) \mid x_{1} \in\left\{-1, M_{1}+1\right\}, x_{2} \in \mathbb{X}_{2}\right\}, \forall u_{b} \in \mathbb{B}, \forall u_{p} \in \mathbb{P}, \forall u_{r} \in \mathbb{R}, \\
& Y_{i, \underline{\mathbf{x}}, u_{b}, u_{p}, u_{r}}=0, \forall i \in \mathbb{E}, \forall \underline{\mathbf{x}} \in\left\{\left(x_{1}, x_{2}\right) \mid x_{1} \in \mathbb{X}_{1}, x_{2} \in\left\{-1, M_{2}+1\right\}\right\}, \forall u_{b} \in \mathbb{B}, \forall u_{p} \in \mathbb{P}, \forall u_{r} \in \mathbb{R}, \\
& Y_{i, \underline{\mathbf{x}}, u_{b}, u_{p}, u_{r}} \geq 0, \forall i \in \mathbb{E}, \forall \underline{\mathbf{x}} \in \mathbb{F}^{\prime}, \forall u_{b} \in \mathbb{B}, \forall u_{p} \in \mathbb{P}, \forall u_{r} \in \mathbb{R} .
\end{aligned}
$$

The objective function defined in Equation (10) is to maximize the long-run average reward of the manufacturer. Constraint (11) is the balance equations that satisfy the flow into state $(i, \underline{\mathbf{x}})$ equals the flow out of state $(i, \underline{\mathbf{x}})$. Since the decision variables constitute a probability mass function, they must be non-negative real numbers and their summation must be equal to 1 . Constraint (17) satisfies the condition on the non-negativity of the decision variables. Constraint (12) ensures that the sum of the decision variables equals to 1 . Constraint (13) stipulates that production is not possible when the raw materials inventory is empty. Constraint (14) ensures that an incoming demand cannot be satisfied if there is no stock in the finished goods inventory. When $x_{1}$ is 0 or $M_{1}$ in the vector $\underline{\mathbf{x}}$, Constraint (11) yields the decision variables that are out of the state space due to $Y_{i, \underline{\mathbf{x}}-e_{1}, u_{b}, u_{p}, u_{r}}$ and $Y_{i, \underline{\mathbf{x}}+e_{1}-e_{2}, u_{b}, u_{p}, u_{r}}$. Constraint (15) eliminates these trivial decision variables from the formulation. Since a similar situation is observed when $x_{2}$ is 0 or $M_{2}$ in the vector $\underline{\mathbf{x}}$, Constraint (16) is also included into the formulation. In another words, Constraints (15) and (16) prevent probability leakage from the outside of the state space.

We analytically investigate the structure of the above linear programming problem by defining the number of decision variables and the number of constraints. Except the nonnegativity constraint, the numbers of variables and constraints are $8 L\left(M_{1}+1\right)\left(M_{2}+1\right)$ and $1+$ $L\left(M_{1}+1\right)\left(M_{2}+1\right)+20 L\left(\left(M_{1}+1\right)+\left(M_{2}+1\right)\right)$, respectively. Since the number of variables and constraints polynomially grow as the size of state space increases, the size of the LP problem mostly stays at a manageable level. We also numerically examined the effect of the size of the state space on the computation time. For the examination, we used GAMS modeling environment with 
CPLEX 12.5 LP solver on a computer that has $2.7 \mathrm{GHz}$ Intel core i5 processor and 8 GB RAM. The results confirmed our analytical findings and showed that the LP formulation manage to find the solution in less than one and half minute when the state space size is less than 2 million. We believe that a model including 2,000,000 states is sufficiently precise to deal with the fluctuating sales and purchasing prices in a production/inventory setting. For instance, this structure allows us to analyze a case in which there exist 400 different sales and purchasing prices combinations and truncation levels are 71 .

\section{Numerical Analysis}

In this section, we examine the effects of variations in the purchasing and sales prices, the correlation between purchasing and sales prices, traffic intensity, and limited inventory capacities on the system performance measures through a range of numerical experiments. Before presenting results, we primarily introduce the long-run measures regarding the dynamics of the operating environment and manufacturing system and justify the chosen parameters.

\subsection{The long-run measures regarding the dynamics of the operating environment and manufacturing system}

In the numerical analysis, we consider the long-run average reward $(\phi)$, customer service level $(\alpha)$, average stock level in the raw materials inventory $\left(\mathrm{E}\left[x_{1}\right]\right)$, and average stock level in the finished goods inventory $\left(\mathrm{E}\left[x_{2}\right]\right)$ as the system performance measures. The definitions of these performance measures are given as follows:

$$
\begin{aligned}
\phi & =\sum_{i \in \mathbb{E}} \sum_{\underline{\mathbf{x}} \in \mathbb{F}^{\prime}} \sum_{u_{b} \in \mathbb{B}} \sum_{u_{p} \in \mathbb{P}} \sum_{u_{r} \in \mathbb{R}} Y_{i, \underline{\mathbf{x}}, u_{b}, u_{p}, u_{r}}\left(\lambda u_{r} s_{i}-\delta u_{b} c_{i}-\mu u_{p} w-x_{1} h_{1}-x_{2} h_{2}\right), \\
\alpha & =\sum_{i \in \mathbb{E}} \sum_{\underline{\mathbf{x}} \in \mathbb{F}^{\prime}} \sum_{u_{b} \in \mathbb{B}} \sum_{u_{p} \in \mathbb{P}} \sum_{u_{r} \in\{1\}} Y_{i, \underline{\mathbf{x}}, u_{b}, u_{p}, u_{r}}, \\
\mathrm{E}\left[x_{1}\right] & =\sum_{i \in \mathbb{E}} \sum_{\underline{\mathbf{x}} \in \mathbb{F}^{\prime}} \sum_{u_{b} \in \mathbb{B}} \sum_{u_{p} \in \mathbb{P}} \sum_{u_{r} \in \mathbb{R}} x_{1} Y_{i, \underline{\mathbf{x}}, u_{b}, u_{p}, u_{r}}, \\
\mathrm{E}\left[x_{2}\right] & =\sum_{i \in \mathbb{E}} \sum_{\underline{\mathbf{x}} \in \mathbb{F}^{\prime}} \sum_{u_{b} \in \mathbb{B}} \sum_{u_{p} \in \mathbb{P}} \sum_{u_{r} \in \mathbb{R}} x_{2} Y_{i, \underline{\mathbf{x}}, u_{b}, u_{p}, u_{r} .}
\end{aligned}
$$

In all the numerical instances, we examine a special setting where the sales and purchasing prices change only at High (H) and Low (L) states. In this setting, the sales prices in the low and high 
states are denoted by $s_{L}$ and $s_{H}$ whereas the purchasing prices in the low and high states are represented as $c_{L}$ and $c_{H}$. To model such a setting with the framework introduced in this study, we map each possible binary combination of the purchasing and sales prices to a certain environment state. So, we define four different environment states and list them in below:

$1=$ Purchasing and sales prices are High $(\mathrm{HH}), c_{1}=c_{H}$ and $s_{1}=s_{H}$, $2=$ Purchasing price is Low and sales price is High (LH), $c_{2}=c_{L}$ and $s_{2}=s_{H}$, $3=$ Purchasing price is High and sales price is Low (HL), $c_{3}=c_{H}$ and $s_{3}=s_{L}$, $4=$ Purchasing and sales prices are Low (LL), $c_{4}=c_{L}$ and $s_{4}=s_{L}$.

With the considered setting, we can characterize the long-run probability distribution associated with the environment states by solving Equations (22) and (23), simultaneously:

$$
\begin{array}{r}
\Pi^{T} Q=0, \\
\Pi 1=1 .
\end{array}
$$

$\Pi^{T}$ is the vector including the long-run probability distribution associated with the environment states, i.e., $\Pi^{T}=\left[\pi_{1} \pi_{2} \pi_{3} \pi_{4}\right]$. With this vector, we define the long-run expectation and variance of the purchasing price as in the following equations and respectively denote them by $\mathrm{E}[C]$ and $\operatorname{Var}[C]:$

$$
\begin{gathered}
\mathrm{E}[C]=c_{H}\left(\pi_{1}+\pi_{3}\right)+c_{L}\left(\pi_{2}+\pi_{4}\right), \\
\operatorname{Var}[C]=\left(c_{H}-c_{L}\right)^{2}\left(\pi_{1}+\pi_{3}\right)\left(\pi_{2}+\pi_{4}\right) .
\end{gathered}
$$

Thus, the coefficient of variation for the purchasing price is defined as $C V_{C}=\sqrt{\operatorname{Var}[C]} / \mathrm{E}[C]$. The long-run expectation and variance of the sales price are respectively defined in below and denoted by $\mathrm{E}[S]$ and $\operatorname{Var}[S]$ :

$$
\begin{gathered}
\mathrm{E}[S]=s_{H}\left(\pi_{1}+\pi_{2}\right)+s_{L}\left(\pi_{3}+\pi_{4}\right), \\
\operatorname{Var}[S]=\left(s_{H}-s_{L}\right)^{2}\left(\pi_{1}+\pi_{2}\right)\left(\pi_{3}+\pi_{4}\right) .
\end{gathered}
$$


With the above expressions, the coefficient of variation for the sales price is $C V_{S}=\sqrt{\operatorname{Var}[S]} / \mathrm{E}[S]$. Additionally, the correlation between purchasing and sales prices, $\rho_{C S}$, is obtained as follows:

$$
\rho_{C S}=\frac{\pi_{4} \pi_{1}-\pi_{2} \pi_{3}}{\sqrt{\left(\pi_{1}+\pi_{3}\right)\left(\pi_{2}+\pi_{4}\right)} \sqrt{\left(\pi_{1}+\pi_{2}\right)\left(\pi_{3}+\pi_{4}\right)}} .
$$

From Equation (28), we observe that when $\pi_{2} \pi_{3}>\pi_{4} \pi_{1}$ there is a negative correlation between purchasing and sales prices. The same equation also implies that for a positive correlation between purchasing and sales prices, $\pi_{2} \pi_{3}<\pi_{4} \pi_{1}$ condition should be satisfied. When $\pi_{2} \pi_{3}=\pi_{4} \pi_{1}$ holds there is no correlation between these two market prices.

The correlation between sales and purchasing prices can only be manipulated with the transition matrix since it is a function of the long-run probability distribution of the environment states. Hence, in the numerical examples, we form the transition matrices in such a way that the specified correlation coefficients are obtained. While forming the transition matrices, we follow a two-step procedure. The procedure allows the correlation coefficient to set the specified level while keeping the expectations and variances of the sales and purchasing prices at the same levels. Thus, for the numerical instances being examined in the same group, we are able to observe the direct effects of the correlation coefficient on the system. If the transition matrices are not formed in this way, the expectations and variances of the sales and purchasing prices change each time the correlation coefficient changes. In such a case, the analysis would yield biased and incorrect results. To initiate the procedure, $\mathrm{E}[C], \operatorname{Var}[C], \mathrm{E}[S], \operatorname{Var}[S], \rho_{C S}, s_{H}, s_{L}, c_{H}, c_{L}$, and the average time spent in each environment state, $T_{i}$ where $i \in\{1,2,3,4\}$, should be given as the input parameters. The details of the procedure are discussed in the Appendix B.

\subsection{Parameter settings}

In the numerical analysis, we study four different scenarios and list the parameters of each scenario in Table 3. In Scenario 1, 2, and 3, we consider a structure where $\delta>\mu>\lambda$ and normalize the demand and raw material arrival rates according to the production rate. With this structure, the manufacturer has a sufficient capacity and number of raw material purchasing opportunities to satisfy the arriving demand. This structure allows us to directly observe the impacts of the 
fluctuating and correlated purchasing and sales prices on the system when Scenario 1, 2, and 3 are considered. To obtain the correlation coefficients considered in the scenarios, we utilize the average amounts of time spent in the environments given in Table 4. One can notice that average time spent in each environment state is typically set to a large value compared to the average interarrival times assigned for the production, demand, and raw material processes. This implies that times to change the environment states are much longer than the ones for the other processes. Hence, the manufacturer is able to deduce and adjust to an environmental change in a relatively short time period.

\begin{tabular}{|c|c|c|c|}
\hline Scenario & Th & $\begin{array}{l}\text { invariant parameters in } \\
\text { the scenario }\end{array}$ & $\begin{array}{c}\text { The changing parameters in } \\
\text { the scenario }\end{array}$ \\
\hline Scenario 1 & $\begin{aligned} \mu & =1.00 \\
h_{1} & =0.04 \\
C V_{C} & =0.10\end{aligned}$ & $\begin{aligned} \delta & =1.50 \quad \lambda=0.80 \quad w=0.10 \\
h_{2} & =0.04 \quad \mathrm{E}[S]=1.80 \quad \mathrm{E}[C]=1.10 \\
c_{H} & =1.20 \quad c_{L}=1.00\end{aligned}$ & $\begin{array}{l}C V_{S} \in\{0.30,0.20,0.10\} \\
s_{H} \in\{2.35,2.20,2.00\}, s_{L} \in\{1.25,1.40,1.55\} \\
\rho_{C S} \in\{-0.90,0.80, \ldots, 0.80,0.90\}\end{array}$ \\
\hline enario 2 & $\begin{aligned} \mu & =1.00 \\
h_{1} & =0.04 \\
C V_{S} & =0.10\end{aligned}$ & $\begin{array}{rrr}\delta & =1.50 \quad \lambda=0.80 \quad w=0.10 \\
h_{2}=0.04 & \mathrm{E}[C]=1.50 & \mathrm{E}[S]=2.40 \\
s_{H}=2.65 \quad s_{L}=2.15\end{array}$ & $\begin{array}{l}C V_{C} \in\{0.30,0.20,0.10\} \\
c_{H} \in\{2.00,1.80,1.65\}, c_{L} \in\{1.00,1.20,1.35\} \\
\rho_{C S} \in\{-0.90,0.80, \ldots, 0.80,0.90\}\end{array}$ \\
\hline Scenario 3 & $\begin{aligned} \mu & =1.00 \\
h_{2} & =0.04 \\
C V_{C} & =0.15 \\
c_{L} & =1.00\end{aligned}$ & $\begin{array}{rrr}\delta=1.50 & w=0.10 & h_{1}=0.04 \\
\mathrm{E}[S] & =1.90 \quad \mathrm{E}[C]=1.20 & C V_{S}=0.20 \\
s_{H} & =2.30 \quad s_{L}=1.50 & c_{H}=1.40\end{array}$ & $\begin{array}{l}\lambda \in\{0.80,0.90, \ldots, 2.50\} \\
\rho_{C S} \in\{-0.60,0,0.60\}\end{array}$ \\
\hline Scenario 4 & $\begin{aligned} h_{1} & =0.04 \\
\mathrm{E}[C] & =1.20 \\
s_{L} & =1.50\end{aligned}$ & $\begin{array}{rrrr}h_{2}=0.04 & w=0.10 & \mathrm{E}[S]=1.90 \\
C V_{S}=0.20 & C V_{C}=0.15 & s_{H}=2.30 \\
c_{H}=1.40 & c_{L}=1.00 & \rho_{C S}=0\end{array}$ & $\begin{array}{l}\lambda \in\{0.60,0.70, \ldots, 2.20\} \\
\mu \in\{1.00,1.60\}, \delta \in\{1.60,1.00\} \\
M_{1}+M_{2} \in\{2,3, \ldots, 10\}\end{array}$ \\
\hline
\end{tabular}

Table 3 The scenarios specified to analyze the effects of the parameters on the system performance measures

In each scenario, we set the long-run averages of purchasing and sales prices in such a way that the relative difference between these two parameters does not exceed $60 \%$. We believe that this structure would provide a better representation for a production/inventory system in practice 
because it avoids the non-realistic environments where the long-run profit margins for the manufacturer are too low or high. While setting the values of the parameters whose impacts on the system would be analyzed, we utilize the specified coefficient of variation for the corresponding parameter and normalize all the market prices according to the lowest purchasing price considered in the corresponding scenario. In all scenarios, we ensure that the lowest sales price is higher than the highest purchasing price. Otherwise, the manufacturer would prefer not to operate in the setting where the sales price is lower than the purchasing price, and so, the results would be trivial. Additionally, in all the scenarios, we consider that the holding costs for the raw material and finished goods inventories equal each other and set them as not-exceeding $4 \%$ of the long-run average of the purchasing prices.

\begin{tabular}{ccccccccccccccccccccccc}
\multicolumn{11}{c|}{$\rho_{C S}$} \\
\cline { 2 - 3 } & -0.9 & -0.8 & -0.7 & -0.6 & -0.5 & -0.4 & -0.3 & -0.2 & -0.1 & 0 & 0.1 & 0.2 & 0.3 & 0.4 & 0.5 & 0.6 & 0.7 & 0.8 & 0.9 \\
\hline$T_{1}$ & 50 & 50 & 50 & 50 & 50 & 50 & 50 & 50 & 50 & 50 & 41 & 33 & 27 & 21 & 17 & 13 & 9 & 6 & 3 \\
$T_{2}$ & 700 & 450 & 283 & 200 & 150 & 117 & 93 & 75 & 61 & 50 & 50 & 50 & 50 & 50 & 50 & 50 & 50 & 50 & 50 \\
$T_{3}$ & 700 & 450 & 283 & 200 & 150 & 117 & 93 & 75 & 61 & 50 & 50 & 50 & 50 & 50 & 50 & 50 & 50 & 50 & 50 \\
$T_{4}$ & 50 & 50 & 50 & 50 & 50 & 50 & 50 & 50 & 50 & 50 & 41 & 33 & 27 & 21 & 17 & 13 & 9 & 6 & 3 \\
\hline
\end{tabular}

Table 4 The average time spent in environment state $i$ for the given correlation coefficient between sales and purchasing prices

From the preliminary numerical analysis results, we have observed that the truncation levels between 20 and 25 are typically sufficiently large to reach the optimal solution for the system parameters used. We have employed this observation while solving the problem instances generated with Scenario 1, 2 and 3. More specifically, we have primarily solved each problem instance by using two different truncation level settings, i.e., $M_{1}=M_{2}=25$ and $M_{1}=M_{2}=26$. Later on, for each problem instance, we have compared the results obtained with these two settings. The comparison results indicate that none of the reward functions in the problem instances improves when we change the truncation levels from 25 to 26 . Accordingly, the setting where $M_{1}=M_{2}=25$ guarantees that we obtain the globally optimal solution for each problem instance in all the scenarios. 




(a)

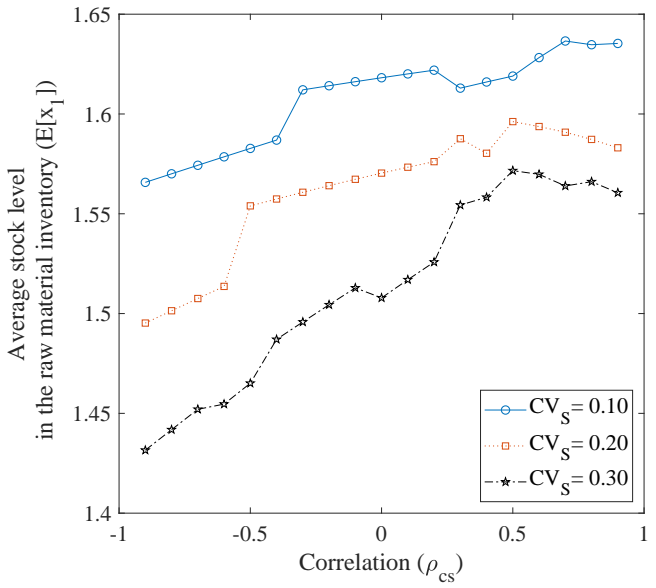

(c)

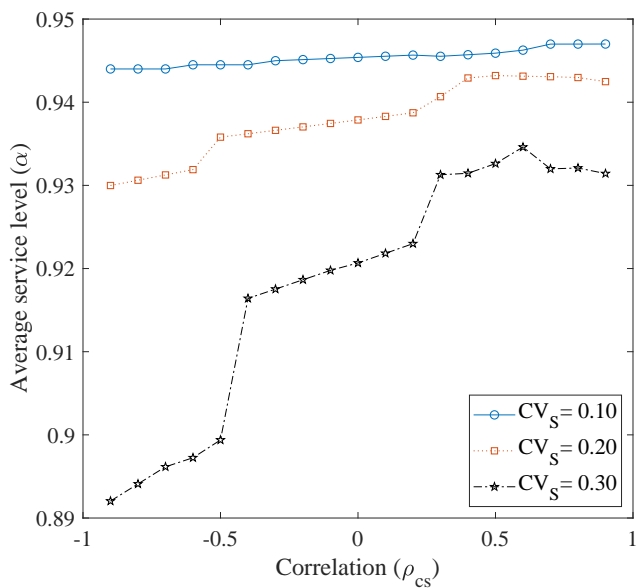

(b)

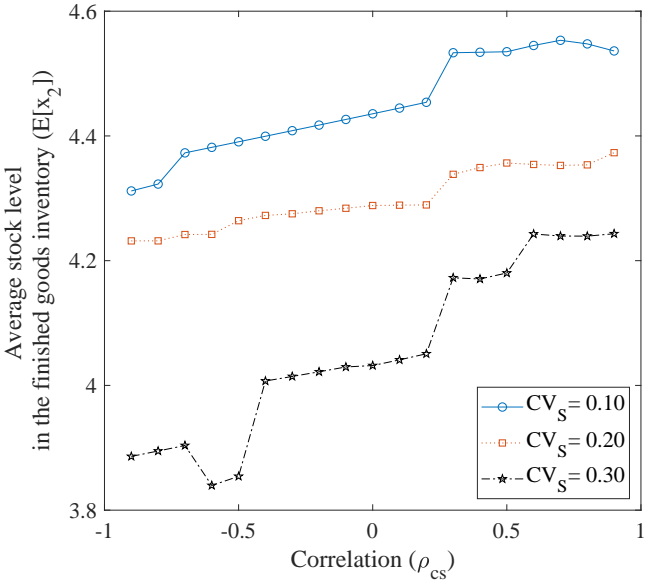

(d)

Figure 4 The impacts of the correlation between sales and purchasing prices and sales price variation on $\mathbf{4}(\mathbf{a})$ the manufacturer's profit, $\mathbf{4 ( b )}$ the average service level, $\mathbf{4 ( c )}$ the average stock level in the raw materials inventory, and $\mathbf{4}(\mathrm{d})$ the average stock level in the finished goods inventory

\subsection{Effects of the correlation between sales and purchasing prices and sales price variation on the system}

To analyze the impacts of the correlation between sales and purchasing prices and sales price variation on the system, we use Scenario 1 given in Table 3 . With this scenario, for each $C V_{S}$ value, we generate 19 problem instances and depict the corresponding result in Figure 4.

Figure 4(a) shows that the manufacturer's profit increases with the sales price variability. When the sales price variability is high the profit margin per unit obtained in state 3 considerably declines. Therefore, in state 3 , the manufacturer reduces the raw material purchases by lowering the threshold values regarding the purchasing decision. So, as Figures 4(c) and 4(d) indicate, the average stock 
levels in the raw materials and finished goods inventories decrease as the sales price variability increases. Figure 4(b) also indicates that the average service level reduces due a decrease in the average stock levels in the raw materials and finished goods inventories. Since the average stock levels in the raw materials and finished goods inventories decrease the manufacturer saves on the purchasing and inventory holding costs. Additionally, the profit margin in state 3 is the lowest one among the other states, because the sales price is low and the purchasing price is high. As a result, the revenue loss due to less stock in state 3 is easily compensated by the sales in the other states and the savings from purchasing and inventory holding costs. These facts reveal the main rationale behind the increase in manufacturer's profit with an increase in sales price variability.

Figure 4(a) also shows that when the correlation between sales and purchasing prices shifts from toward -0.90 to 0.90 the manufacturer's profit decreases. In the case where the correlation between sales and purchasing prices is negative, the manufacturer reduces the raw material purchases in state 3 and saves on the purchasing and inventory holding costs. Figure 4(a) also implies that the amount of savings obtained in the negative correlation case increases as the variability of sales price increases. Lowering the raw material purchases in state 3 yields a decrease in the average service level, average stock level in the raw materials inventory, and average stock level in the finished goods inventory (see Figures 4(b), 4(c), and 4(d)). However, when the correlation proceeds from the negative values to the positive values the manufacturer starts to actively use four environment states. Hence, when the correlation is positive we observe an increase in the average service level, average stock level in the raw materials inventory, and average stock level in the finished goods inventory. An increase in these measures leads to a decrease in the amount of savings obtained from purchasing and inventory holding costs so that the manufacturer's profit reduces.

\subsection{Effects of the correlation between sales and purchasing prices and purchasing price variation on the system}

To analyze the impacts of the correlation between sales and purchasing prices and purchasing price variation on the system, we employ Scenario 2 given in Table 3 . With this scenario, for each $C V_{C}$ value, we generate 19 problem instances and depict the corresponding result in Figure 5. 


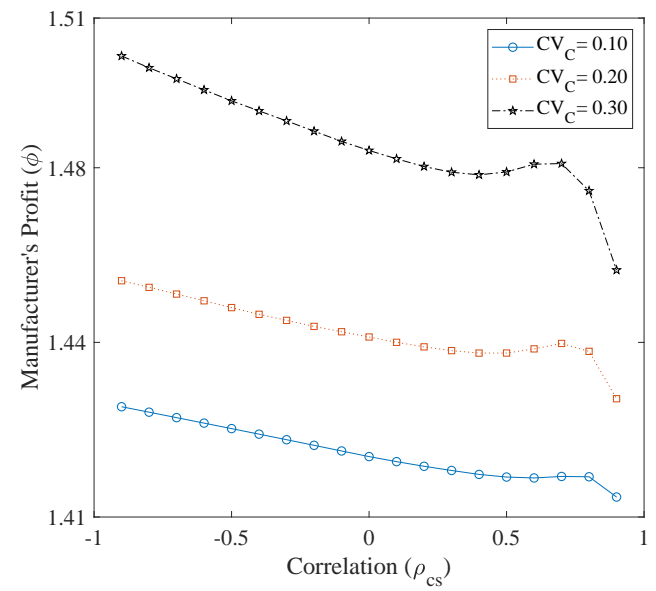

(a)

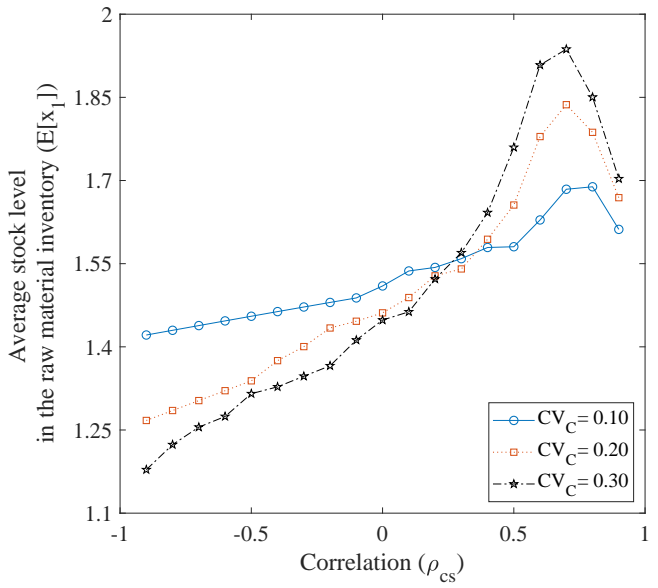

(c)

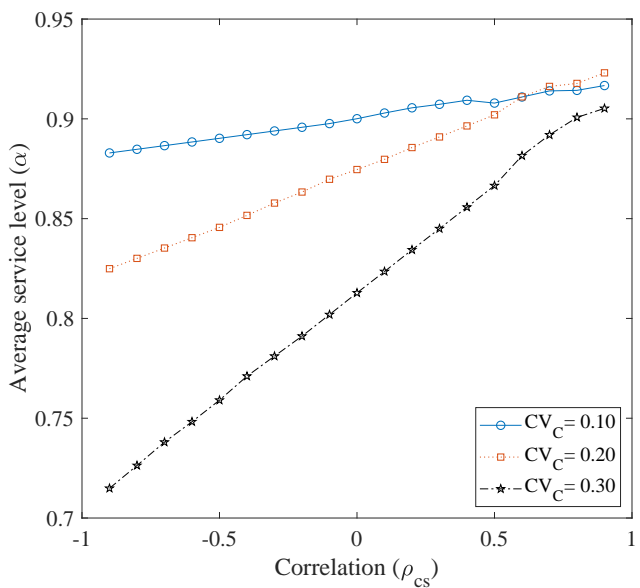

(b)

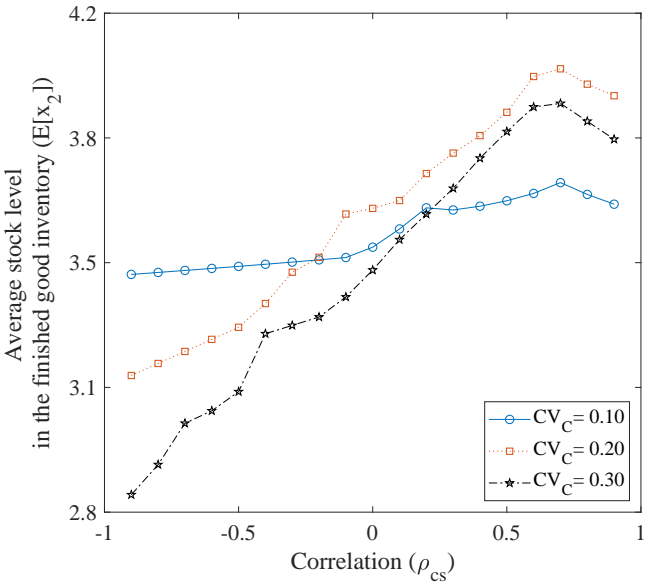

(d)

Figure 5 The impacts of the correlation between sales and purchasing prices and purchasing price variation on $\mathbf{5}(\mathbf{a})$ the manufacturer's profit, $\mathbf{5}(\mathbf{b})$ the average service level, $\mathbf{5}$ (c) the average stock level in the raw materials inventory, and $\mathbf{5}(\mathbf{d})$ the average stock level in the finished goods inventory

Figure 5(a) indicates that the manufacturer's profit decreases when we shift the correlation between purchasing and sales prices from toward -0.90 to 0.90 . When there is a negative correlation between sales and purchasing prices the manufacturer reduces the raw material purchases in state 3. Figures $5(\mathrm{c})$ and $5(\mathrm{~d})$ show that reducing the raw material purchases in state 3 declines the average stock levels in the raw materials and finished goods inventories. With this policy, the manufacturer saves on the purchasing and inventory holding costs and increases his profit. Figure 5(a) shows that the amount of savings increases as the variation of the purchasing price rises. In addition, since the average stock levels in the raw materials and finished goods inventories decrease in the case where 
the correlation between sales and purchasing prices is negative, the average service level declines (see Figure 5(b)).

When the correlation between purchasing and sales prices shifts from toward the negative values to the positive values the manufacturer considerably increases the raw material purchases in state 2. Since the purchasing price is low in state 2 the manufacturer aims to save on the purchasing costs by implementing this policy. Figure 5(a) shows that the amount of this saving slightly rises when the purchasing price variation is high and the correlation coefficient is between 0.4 and 0.7 . In Figures 5(c) and 5(d), we also observe that this policy causes a significant increase in the average stock levels in the raw materials and finished goods inventories when the correlation coefficient is between 0.4 and 0.7 . Figure $5(\mathrm{~b})$ indicates that since the average stock levels in both inventories increases with the positive correlation coefficient the average service level rises. The manufacturer could not raise the raw material stocks to the level that he aims because the average time spent in state 2 notably reduces when the correlation coefficient is greater 0.7 . Hence, we observe that when the correlation coefficient is greater 0.7 the manufacturer's profit, average stock level in the raw materials inventory, and average stock level in the finished goods inventory decrease (see Figures $5(\mathrm{a}), 5(\mathrm{c})$, and $5(\mathrm{~d}))$.

\subsection{Effects of the correlation between sales and purchasing prices and traffic intensity on the system}

To examine the effects of the correlation between sales and purchasing prices and traffic intensity on the system, we utilize Scenario 3 given in Table 3 . With this scenario, for each $\rho_{C S}$ value, we generate 18 problem instances and depict the corresponding result in Figure 6.

Figure 6(a) shows that the manufacturer's profit increases when the demand arrival rate raises. An increase in the demand arrival rate yields a higher number of customers arriving at the system. To satisfy the increasing demand, the manufacturer increases the raw material purchases. Hence, in Figure 6(c), we notice that the average stock level in the raw materials inventory rises when the demand arrival rate increases. In Figure 6(d), we observe that since the manufacturer's production capacity is limited the average stock level in the finished goods inventory decreases with an increase 


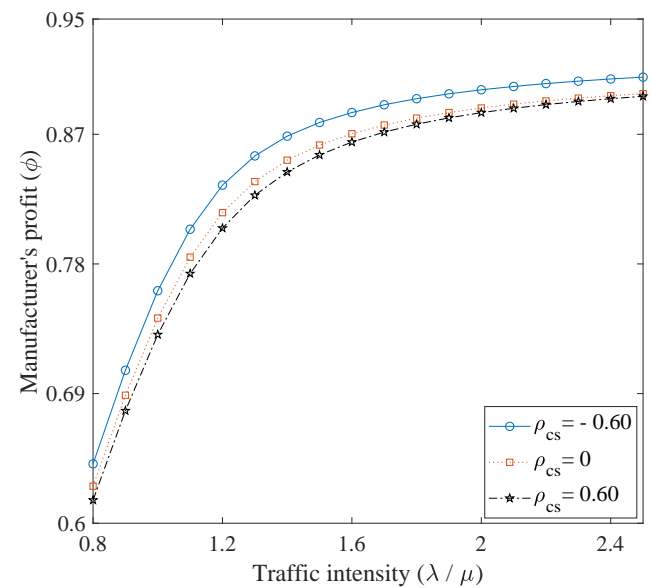

(a)

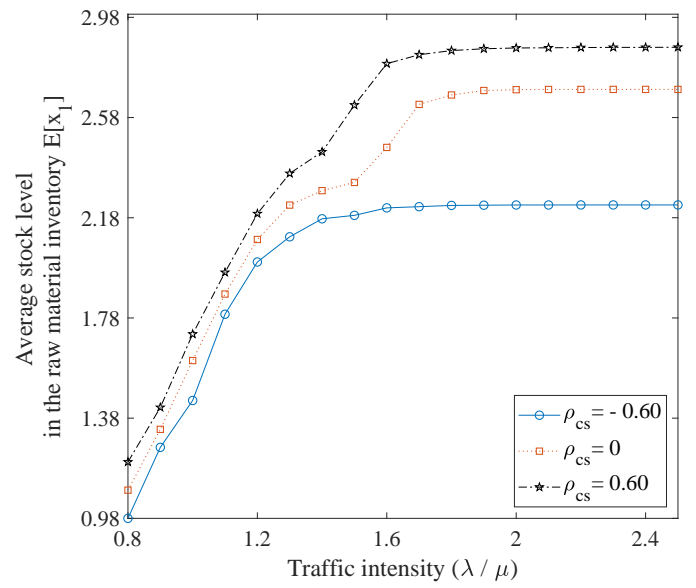

(c)

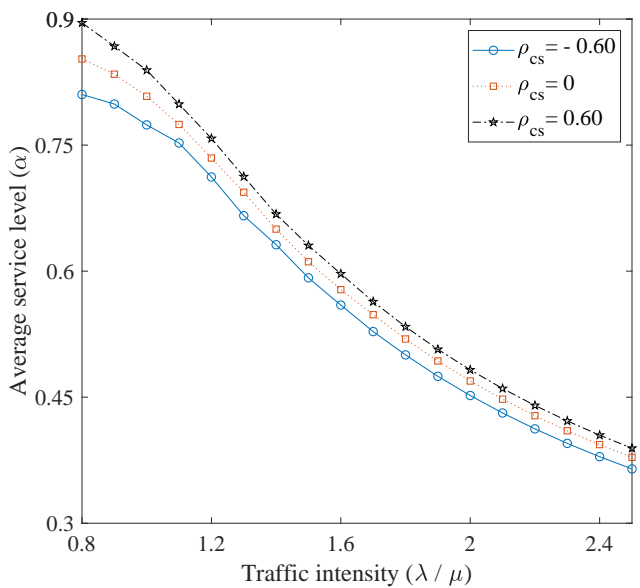

(b)

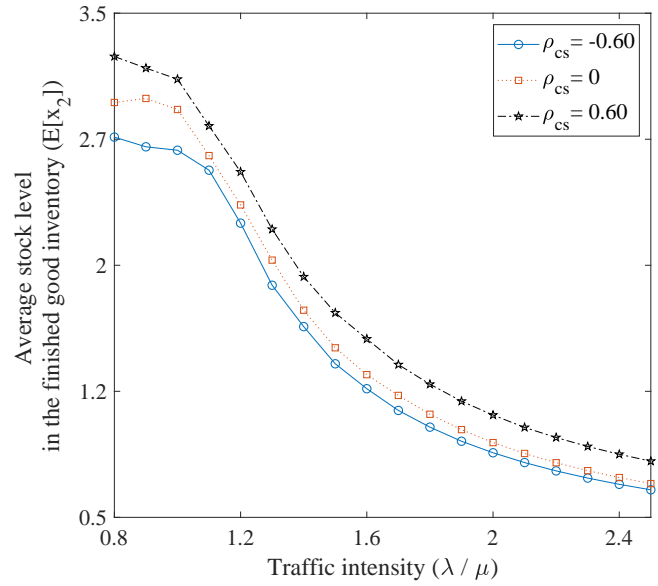

(d)

Figure 6 The impacts of the correlation between sales and purchasing prices and traffic intensity on $\mathbf{6}(\mathbf{a})$ the manufacturer's profit, $\mathbf{6}(\mathbf{b})$ the average service level, $\mathbf{6}(\mathbf{c})$ the average stock level in the raw materials inventory, and $\mathbf{6}(\mathbf{d})$ the average stock level in the finished goods inventory

in the demand arrival rate. As Figure 6(b) shows, the decreasing average stock level in the finished goods inventory due to an increase in the demand arrival rate yields a decrease in the average service level.

Figure 6(a) also indicates that the negative correlation between sales and purchasing prices leads to a slight increase in the manufacturer's profit. For each different correlation coefficient, the other performance measures, the average service level, average stock level in the raw materials inventory, and average stock level in the finished goods inventory, almost follow a similar pattern (see Figures 6(b), 6(c), and 6(d)). So, we conclude that the impacts of limited production and supply capacities 
on the system dominates the impact of the correlation between purchasing and sales prices on the system.

\subsection{Effects of limited finished goods and raw materials inventories on the system}

In practice, the manufacturer can restrict the capacities of the finished goods and raw materials inventories due to limited financial and physical resources. In such a case, the manufacturer must foresee the effects of the limited finished goods and raw materials inventories on the system and decides how to allocate the limited capacity to the inventories so as to maximize the profit. By considering this fact, in this section, we discuss how the capacitated finished goods and raw materials inventories affect the system.

To analyze the impacts of limited finished goods and raw materials inventories on the system, we utilize Scenario 4 given in Table 3. For each combination of given production, raw material arrival, and demand arrival rates, we generate a set of problem instances by ranging the sum of $M_{1}$ and $M_{2}$ from 2 to 10 . With this setting, we limit the capacities of the finished goods and raw materials inventories and obtain 306 problem instances for both $\mu<\delta$ and $\mu>\delta$ cases. For each given $M_{1}+M_{2}$ value in a particular problem set, we obtain how the manufacturer assigns its limited capacity to the finished goods and raw material inventories by optimizing the profit. Thus, in each problem set, we are able to calculate the averages of the capacities allocated to the raw materials and finished goods inventories. We depict these results in Figure 7.

Figure 7(a) shows that the manufacturer allocates more capacity to the finished goods inventory than to the raw materials inventory when $\lambda<\mu<\delta$ holds. Figure 7 (a) also indicates that as the demand rate increases the average amount of capacity allocated to the raw materials inventory increases. With an increase in the demand arrival rate, the production and raw material processes relatively slow down. This fact forces the manufacturers to hedge against a starvation risk in the raw materials inventory. As a result, the average amount of capacity allocated to the raw materials inventory increases whereas the average amount of capacity allocated to the finished goods inventory decreases. 




(a)

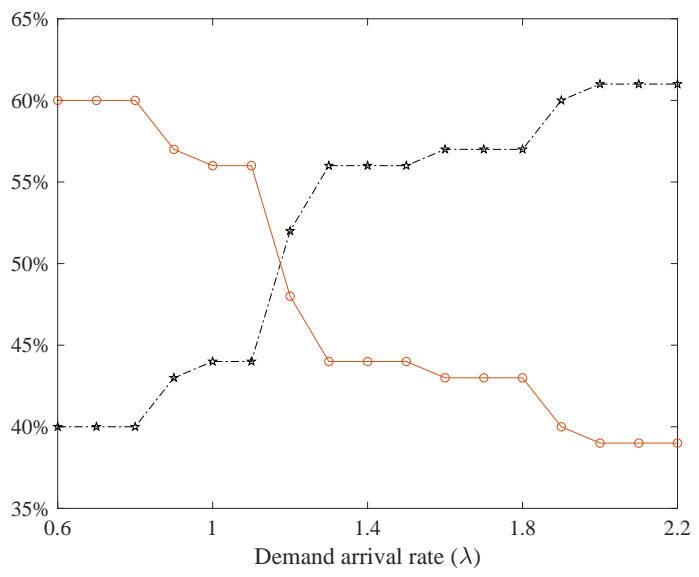

(b)

Average percentage of total capacity __ Average percentage of total capacity assigned to the raw material inventory assigned to the finished good inventory

Figure 7 The averages of the percentages for total capacity assigned to the raw materials and finished goods inventories $\mathbf{7}(\mathbf{a})$ when $\mu=1.00<\delta=1.60 \mathbf{7}(\mathbf{b})$ when $\mu=1.60>\delta=1.00$

Figure 7(b) implies that the manufacturer allocates a higher capacity to the finished goods inventory up to the point where $\delta=\lambda=1.20$. At the point where $\delta=\lambda=1.20$, the average amount of capacity allocated to the raw materials inventory almost equals the average amount of capacity allocated to the finished goods inventory. Figure $7(\mathrm{~b})$ shows that when the demand arrival rate is greater than 1.20 the average amount of capacity allocated to the raw materials inventory becomes to be higher than the average amount of capacity allocated to the finished goods inventory. The main rationale behind this trend is similar to that observed in the first version of the basic problem. When the demand arrival rate is greater than 1.20, the purchasing process would be the bottleneck of the system because of having the smallest rate compared to the demand arrival and production rates. This fact forces the manufacturer to avoid a starvation risk in the raw materials inventory. Consequently, an increase in the demand arrival rate leads to an increase in the average amount of capacity allocated to the raw materials inventory and a decrease in the average amount of capacity allocated to the finished goods inventory. With this analysis, we conclude that the manufacturer gives a priority to increase the capacity of inventory that is close to the system's bottleneck in terms of rates. 
The impacts of limited finished goods and raw materials inventories on the system are also examined with the sales price variation, purchasing price variation, and correlation between sales and purchasing prices. However, we observe that the impacts of limited finished goods and raw materials inventories on the system are not affected by the sales price variation, purchasing price variation, and correlation between sales and purchasing prices. Due to the sake of clarity, we do not include these analyses in the current work.

\subsection{Benefits of using the optimal policy to the profit when compared to the Buy Low and Sell High policy}

The manufacturers which are subject to the random fluctuations in purchasing and sales prices may be inclined to adopt the Buy Low and Sell High naive policy where the manufacturer buys the raw materials only when the purchasing price is low and sells the finished goods only when the sales price is high. Although it is very easy to implement this policy, the naive policy leads to a profit loss for the manufacturer. By considering this fact, in this section, we discuss how much the manufacturer can improve the profit by using the optimal policy instead of using the naive one.

For the discussion, we consider Scenario 1, 2, and 3 given in Table 3. As done in the previous sections, the optimal control policies for the considered scenarios are numerically obtained by using the linear programming formulation introduced in Equations (10) - (17). In addition, the naive policies for the considered scenarios are numerically derived by adding two extra constraints to the original linear programming formulation. These two constraints are presented as follows:

$$
\begin{aligned}
& Y_{i, \underline{\mathbf{x}}, u_{b}, u_{p}, u_{r}}=0, \forall i \in\{1,3\}, \forall \underline{\mathbf{x}} \in \mathbb{F}^{\prime}, u_{b} \in\{1\}, \forall u_{p} \in \mathbb{P}, \forall u_{r} \in \mathbb{R}, \\
& Y_{i, \underline{\mathbf{x}}, u_{b}, u_{p}, u_{r}}=0, \forall i \in\{3,4\}, \forall \underline{\mathbf{x}} \in \mathbb{F}^{\prime}, u_{b} \in \mathbb{B}, \forall u_{p} \in \mathbb{P}, \forall u_{r} \in\{1\} .
\end{aligned}
$$

Equation (29) implies that the manufacturer buys the raw materials only when the purchasing price is low. Equation (30) stipulates that the manufacturer sells the finished goods only when the sales price is high. For each scenario, we obtain the relative difference between the manufacturer's

profit obtained with the use of the optimal policy and the manufacturer's profit obtained with the use of the naive policy. The results regarding Scenario 1, 2, and 3 are respectively depicted 


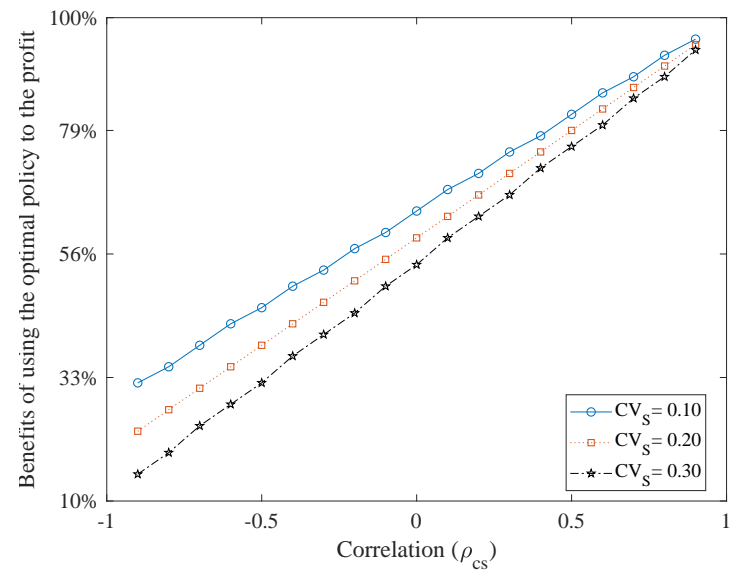

(a)



(b)

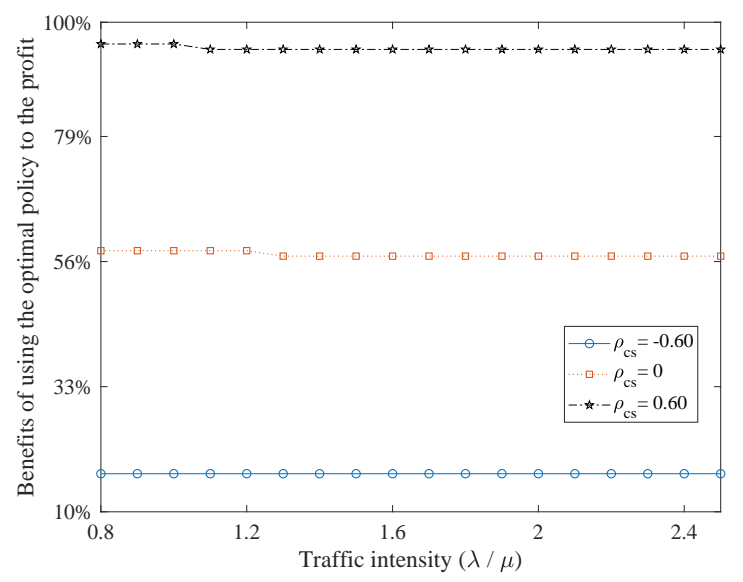

(c)

Figure 8 The benefit obtained by using the optimal policy instead of the naive policy $\mathbf{8}(\mathbf{a})$ when Scenario 1 is considered 8(b) when Scenario 2 is considered 8(c) when Scenario 3 is considered

in Figures 8(a), 8(b), and 8(c). In the figures, a higher difference means that the manufacturer obtains a higher profit by using the optimal policy.

Figures $8(\mathrm{a})$ and $8(\mathrm{~b})$ show that the positive impact of using the optimal policy on the profit decreases when the correlation between sales and purchasing prices shifts from toward 0.90 to -0.90. In the setting where the correlation coefficient is negative, the manufacturer that follows the optimal policy reduces the raw material purchases in state 3 to save on the purchasing and inventory holding costs. Thus, the optimal policy shows a notable similarity to the naive policy in which the manufacturer halts the raw material purchases in state 3 . The similarity that emerges 
between the naive and optimal policies with the negative correlation coefficient leads to a decrease in the positive impact of using the optimal policy on the profit.

Figure 8(a) indicates that an increase in the sales price variation yields a decrease in the positive impact of using the optimal policy on the profit. The main rationale behind this trend is similar to that observed in the negative correlation case. The manufacturer that follows the optimal policy reduces the raw material purchases in state 3 with an increase in the sales price variation. This result implies that as the sales price variation increases the optimal policy resembles the naive policy in which the manufacturer stops purchasing the raw materials in state 3 . The resemblance that emerges between the naive and optimal policies with the high sales price variation yields a decrease in the positive impact of using the optimal policy on the profit. In addition, Figure 8(b) shows that the positive impact of using the optimal policy on the profit decreases with an increase in the purchasing price variation. The main reason behind this trend is similar to that observed in the negative correlation and high sales price variation cases. Figure $8(\mathrm{c})$ implies that there is no significant impact of the traffic intensity rate on the difference between the manufacturer's profit obtained with the use of the naive policy and the manufacturer's profit obtained with the use of the optimal policy.

\subsection{Managerial insights}

In this section, we summarize our key findings that provide managerial insights into how the sales price variation, purchasing price variation, correlation between sales and purchasing prices, limited raw materials and finished goods inventories, and limited production capacity affect the considered system. We also give an insight into under which circumstances the use of the optimal policy significantly improves the profit when compared to the use of the naive policy in which the manufacturer buys only when the purchasing price is low and sells only when the sales price is high. A detailed list regarding these managerial insights is given below:

1. The negative correlation between sales and purchasing prices yields an increase in the manufacturer's profit. In the negative correlation, the manufacturer lowers the average stock levels in 
the raw materials and finished goods inventories. Therefore, the average service level decreases. Decreasing average stock levels in the raw materials and finished goods inventories yield a saving in the purchasing and inventory holding costs. The amount of saving obtained due to the negative correlation leads to an increase in the manufacturer's profit.

2. The positive correlation between sales and purchasing prices leads to a decrease in the manufacturer's profit. When the sales and purchasing prices are positively correlated, the manufacturer increases the average stock levels in the raw materials and finished goods inventories. Therefore, the average service level increases. Increasing the stock levels in the raw materials and finished goods inventories yield higher purchasing and inventory holding costs. This fact results a decrease in the manufacturer's profit when the correlation is positive.

3. The manufacturer's profit rises with an increase in the sales price variation. The main rationale behind this fact is that the increasing sales price variation allows the manufacturer to achieve a significant amount of savings in the purchasing and holding costs. As a result of this fact, when the correlation is negative and the sales price is high the manufacturer obtains the highest profit in the numerical instances.

4. The manufacturer's profit rises with an increase in the purchasing price variation. The main rationale behind this fact is similar to that observed in the negative correlation and high sales price variation cases. Specifically, when the purchasing price variation is high the manufacturer follows a policy that increases the profit by achieving a saving in the purchasing and inventory holding costs.

5. The manufacturer's profit increases as the demand arrival rate increases. With an increase in the demand arrival rate, the number of customers arriving at the system per unit time rises. As a result, the revenue obtained from the sales rises thereby increasing the profit. To satisfy the increasing demand, the manufacturer raises the average stock level in the raw materials inventory. However, due to the limited production capacity, the average stock level in the finished goods inventory decreases with an increase in the demand arrival rate. 
6. When the manufacturer has a limited capacity for assigning both raw materials and finished goods inventories, if the demand arrival rate is the smallest one between the raw material arrival and production rates, increasing the capacity of the finished goods inventory provides a higher profit for the manufacturer. If the raw material arrival rate is the smallest one between the demand arrival and production rates, increasing the capacity of the raw materials inventory yields a higher profit for the manufacturer. By following this policy, the manufacturer aims to avoid starvation that could arise in the inventory that is close to the bottleneck of the system.

7. The negative correlation between the sales and purchasing prices leads to a notable decrease in the difference between the profits achieved by using the optimal and naive policies. An increase either in the sales price variation or in the purchasing price variation also yields a decrease in the positive impact of using the optimal policy on the profit. These observations can be explained by the fact that the high sales price variation, high purchasing price variation, and negative correlation force the manufacturer to follow a policy that is similar to the naive policy.

\section{Conclusion}

We analyze the purchasing, production, and sales policies for a continuous-review discrete material flow production/inventory system with fluctuating and correlated purchasing and sales prices, exponentially distributed raw material and demand inter-arrival times, and processing time. The sales and purchasing prices are driven by the random environmental changes that evolve according to a discrete state space continuous-time Markov process. We model the system as an infinitehorizon Markov decision process under the average reward criterion.

We prove that the optimal purchasing, production, and sales strategies are state-dependent threshold policies. To numerically determine the optimal threshold levels and to validate the analytic results, we propose a solution procedure based on a linear programming formulation. We investigate the effects of sales price variation, purchasing price variation, correlation between sales and purchasing prices, limited raw materials and finished goods inventories, and limited production 
capacity on the system performance measures, through a range of numerical experiments. Based on the numerical results, we provide managerial insights regarding the effects of these parameters on the system.

The negative correlation between the sales and purchasing prices, high sales price variation, and high purchasing price variation leads to an increase in the profit. The main rationale behind the increase in the profit is that these parameter specifications allow the manufacturer to save on the purchasing and inventory holding costs. When the capacity that can be assigned to the raw materials and the finished goods inventories is limited, if the raw materials arrival rate is the smallest compared to the production and the demand arrival rates, increasing the capacity of the raw materials inventory yields a higher profit for the system. However, if the demand arrival rate is the smallest, increasing the capacity of the finished good inventory yields a higher profit for the system. By using this policy, the manufacturer avoids starvation in the inventory that is close to the system's bottleneck.

We examine under which circumstances the use of the optimal policy considerably improves the profit compared to the use of the naive buy low and sell high policy where the manufacturer buys only when the purchasing price is low and sells only when the sales price is high. The negative correlation between sales and purchasing prices, high sales price variation, and high purchasing price variation lead to a decrease in the positive impact of using the optimal policy on the profit. The main rationale behind this result is that the optimal policies derived with these parameter specifications resemblance the naive policy.

This study can be extended in several ways. In this study, we use exponentially distributed raw material and demand arrival rates and processing time. Markov arrival processes can be used to model correlated arrivals and service times. Second, this work provides the basic perspective for the optimal purchasing, production, and sales policies by considering a setting where the manufacturer produces a single product with a reliable machine. Studying the problem with multiple products and unreliable machine assumptions could be an interesting topic for future research. Third, this 
work can be extended by incorporating the pricing decision into the model. Extending this work to a setting with pricing decision would allow us to examine the impacts of different pricing strategies on the system.

\section{Appendix A:}

While deriving the optimal control policies for the purchasing, production, and sales decisions, we use a general framework that is proposed by Koole (2004) to derive convexity results and related properties on the value functions. To able to use this framework in our study, we first convert the manufacturer's maximization problem into an equivalent minimization problem as follows:

$$
v^{\prime}(i, \underline{\mathbf{x}})+g^{*}=\delta T_{b}^{\prime} v^{\prime}(i, \underline{\mathbf{x}})+\mu T_{p}^{\prime} v^{\prime}(i, \underline{\mathbf{x}})+\lambda T_{r}^{\prime} v^{\prime}(i, \underline{\mathbf{x}})+T_{e} v^{\prime}(i, \underline{\mathbf{x}})+h \underline{\mathbf{x}},
$$

where $v^{\prime}(i, \underline{\mathbf{x}})=-v(i, \underline{\mathbf{x}})$. The operators $T_{b}^{\prime} f(i, \underline{\mathbf{x}}), T_{p}^{\prime} f(i, \underline{\mathbf{x}})$, and $T_{r}^{\prime} f(i, \underline{\mathbf{x}})$ for any real-valued function $f(i, \underline{\mathbf{x}})$ are

$$
\begin{aligned}
& T_{b}^{\prime} f(i, \underline{\mathbf{x}})=\min \left\{f\left(i, \underline{\mathbf{x}}+e_{1}\right)+c_{i}, f(i, \underline{\mathbf{x}})\right\}, \\
& T_{p}^{\prime} f(i, \underline{\mathbf{x}})= \begin{cases}\min \left\{f\left(i, \underline{\mathbf{x}}-e_{1}+e_{2}\right)+w, f(i, \underline{\mathbf{x}})\right\}, & x_{1}>0, \\
f(i, \underline{\mathbf{x}}), & x_{1}=0,\end{cases} \\
& T_{r}^{\prime} f(i, \underline{\mathbf{x}})= \begin{cases}\min \left\{f\left(i, \underline{\mathbf{x}}-e_{2}\right)-s_{i}, f(i, \underline{\mathbf{x}})\right\}, & x_{2}>0, \\
f(i, \underline{\mathbf{x}}), & x_{2}=0 .\end{cases}
\end{aligned}
$$

With the modified version of the problem, we consider that the optimal value function satisfies a certain property for all states as specified in the following paragraph. Let $\mathcal{V}$ be a set of real-valued functions defined on $\mathbb{Z}^{3}$ such that if $v^{\prime}(i, \underline{\mathbf{x}}) \in \mathcal{V}$, then

$$
v^{\prime}(i, \underline{\mathbf{x}})+v^{\prime}\left(i, \underline{\mathbf{x}}+d+d^{\prime}\right) \leq v^{\prime}(i, \underline{\mathbf{x}}+d)+v^{\prime}\left(i, \underline{\mathbf{x}}+d^{\prime}\right),
$$

for all $\underline{\mathbf{x}} \in \mathbb{Z}^{2}$ and $d, d^{\prime} \in \mathcal{D}=\left\{e_{1}, e_{2}-e_{1},-e_{2}\right\}$ with $d \neq d^{\prime}$. The inequality defined in Equation (35) implies the multimodularity with respect to both $x_{1}$ and $x_{2}$.

In Lemma 1, we extend the relation between multimodularity and component-wise convexity.

Lemma 1. If $v^{\prime}(i, \underline{\mathbf{x}}) \in \mathcal{V}$, then it also satisfies the inequality below:

$$
2 v^{\prime}\left(i, \underline{\mathbf{x}}+e_{k}\right) \leq v^{\prime}(i, \underline{\mathbf{x}})+v^{\prime}\left(i, \underline{\mathbf{x}}+2 e_{k}\right), \forall \underline{\mathbf{x}} \in \mathbb{Z}^{2} .
$$


Lemma 1 implies that if $v^{\prime}(i, \underline{\mathbf{x}})$ satisfies the inequalities for the multimodularity, it is evidently componentwise convex. The proof related to this result is given in below.

Proof of Lemma 1: When any real-valued function $f(x)$ defined on $\mathbb{N}^{2}$ satisfies the inequalities for the multimodularity $(M M)$, it evidently satisfies the inequalities for both the superconvexity $(S u p e r C)$ and supermodularity (Super). Because Super $C$ and Super consist of exactly the same equations with the $M M$. For detailed definitions of SuperC and Super, we refer to reader the work of Koole (2004). In particular, this proposition implies the following relationship:

$$
M M=\text { SuperC } \cap \text { Super. }
$$

In addition, any real-valued function $f(x)$ defined on $\mathbb{N}^{2}$ satisfying the inequalities SuperC and Super is also component-wise convex $(C C)$. Mathematically, it can be written as follows:

$$
\text { SuperC } \cap \text { Super } \subset C C \text {. }
$$

As a result, we can obtain Lemma 1 by combining these two results presented in above. Specifically, for a fixed environment state $i$, if any real-valued function $v(i, \underline{\mathbf{x}})$ defined on $\mathbb{N}^{3}$ is multimodular with respect to $x_{1}$ and $x_{2}$, then it is also component-wise convex with respect to $x_{1}$ and $x_{2}$. Mathematically, this relationship can be shown as follows:

$$
M M=\text { Super } C \cap S \text { Suer } \subset C C \text {. }
$$

Q.E.D.

In Lemma 2, the relation between multimodularity and joint convexity is described.

Lemma 2. If $v^{\prime}(i, \underline{\mathbf{x}}) \in \mathcal{V}$, then it is jointly convex with respect to both $x_{1}$ and $x_{2}$.

Proof of Lemma 2: Lemma 2 is a direct result obtained from the work of Altman et al. (2000). The author proves that if any real-valued function $f(x)$ defined on $\mathbb{N}^{m}$ is multimodular, then it is evidently jointly convex. For more detailed discussion, we refer to reader the work of Altman et al. (2000).

Q.E.D.

Lemma 3 establishes that for each environment $i$, the operator $T$ preserves multimodularity, componentwise convexity, and joint convexity properties with respect to $x_{1}$ and $x_{2}$.

Lemma 3. If $v^{\prime}(i, \underline{\mathbf{x}}) \in \mathcal{V}$, then $T v^{\prime}(i, \underline{\mathbf{x}}) \in \mathcal{V}$ where $T v^{\prime}(i, \underline{\mathbf{x}})=\delta T_{b}^{\prime} v^{\prime}(i, \underline{\mathbf{x}})+\mu T_{p}^{\prime} v^{\prime}(i, \underline{\mathbf{x}})+\lambda T_{r}^{\prime} v^{\prime}(i, \underline{\mathbf{x}})+$ $T_{e} v^{\prime}(i, \underline{\mathbf{x}})+h \underline{\mathbf{x}}$. Furthermore, $T v^{\prime}(i, \underline{\mathbf{x}}) \in \mathcal{V}$ also preserves the properties proposed in Lemma 1 and Lemma 2. 
Proof of Lemma 3: The operators, $T_{b}^{\prime} f(i, \underline{\mathbf{x}}), T_{p}^{\prime} f(i, \underline{\mathbf{x}})$, and $T_{r}^{\prime} f(i, \underline{\mathbf{x}})$ are variations to the operators introduced in the work of Koole (2004). In his work, it is already shown that these operators preserve the multimodularity and component-wise convexity when any real-valued function $f(i, \underline{\mathbf{x}})$ is multimodular. For more detailed discussion, we refer to reader the work of Koole (2004). The operator described for the environment transitions, $T_{e} f(i, \underline{\mathbf{x}})$, is just a linear combination of any real-valued function $f(i, \underline{\mathbf{x}})$. By assumption, the holding cost is defined as linear. With these facts, we can conclude that the operator $T$ where $T v^{\prime}(i, \underline{\mathbf{x}})=\delta T_{b}^{\prime} v^{\prime}(i, \underline{\mathbf{x}})+\mu T_{p}^{\prime} v^{\prime}(i, \underline{\mathbf{x}})+\lambda T_{r}^{\prime} v^{\prime}(i, \underline{\mathbf{x}})+T_{e} v^{\prime}(i, \underline{\mathbf{x}})+h \underline{\mathbf{x}}$ preserves the multimodularity, joint convexity, and component-wise convexity. Because, it is a linear combination of the operators that preserve the multimodularity, joint convexity, and component-wise convexity.

Q.E.D.

Lemma 3 implies by the value iteration principle that the optimal value function $v^{\prime *}(i, \underline{\mathbf{x}})$ is jointly convex and satisfies the inequalities for component-wise convexity. Since the optimal value function satisfies both joint and component-wise convex and the event operators preserve these convexity properties, there exists a corresponding threshold level for purchasing, production, and sales decisions. Hence, we are able to give the proofs that establish the optimality of threshold policies for purchasing, production, and sales decisions.

Proof of Theorem 1: Let $v^{\prime *}(i, \underline{\mathbf{x}}) \in V$. Due to Lemma 1, we can say that $v^{\prime *}(i, \underline{\mathbf{x}})$ is convex with respect to $x_{1}$ for each $i$ and $x_{2}$, i.e.,

$$
v^{\prime *}\left(i, \underline{\mathbf{x}}+e_{1}\right)-v^{\prime *}(i, \underline{\mathbf{x}}) \geq v^{\prime *}(i, \underline{\mathbf{x}})-v^{\prime *}\left(i, \underline{\mathbf{x}}-e_{1}\right) .
$$

If it is optimal to buy in a state $(i, \underline{\mathbf{x}})$, from Equation (32) we have:

$$
v^{\prime *}\left(i, \underline{\mathbf{x}}+e_{1}\right)+c_{i}<v^{\prime *}(i, \underline{\mathbf{x}}) \Longleftrightarrow v^{\prime *}\left(i, \underline{\mathbf{x}}+e_{1}\right)-v^{\prime *}(i, \underline{\mathbf{x}})<-c_{i} .
$$

Then, by convexity, we have:

$$
-c_{i}>v^{\prime *}\left(i, \underline{\mathbf{x}}+e_{1}\right)-v^{\prime *}(i, \underline{\mathbf{x}}) \geq v^{\prime *}(i, \underline{\mathbf{x}})-v^{\prime *}\left(i, \underline{\mathbf{x}}-e_{1}\right),
$$

implying that it has to be optimal to buy in state $\left(i, \underline{\mathbf{x}}-e_{1}\right)$, as well. Therefore, whenever an optimal policy is to buy in a state $(i, \underline{\mathbf{x}})$ where $\underline{\mathbf{x}}=\left(x_{1}, x_{2}\right)$, it is optimal to buy in all states $(i, \underline{\widetilde{\mathbf{x}}})$ where $\underline{\widetilde{\mathbf{x}}}=\left(\widetilde{x}_{1}, x_{2}\right)$ and $\widetilde{x}_{1}<x_{1}$. A similar discussion can also be adapted to show that if an optimal policy does not buy in a state $(i, \underline{\mathbf{x}})$ where $\underline{\mathbf{x}}=\left(x_{1}, x_{2}\right)$, it continues not to buy in all states $(i, \underline{\widehat{\mathbf{x}}})$ where $\underline{\hat{\mathbf{x}}}=\left(\widehat{x}_{1}, x_{2}\right)$ and $\widehat{x}_{1} \geq x_{1}$. These two statements together imply the existence of an optimal purchasing threshold level. Specifically, for any given state $\left(i^{\prime}, \underline{\mathbf{x}}^{\prime}\right)$ where $\left(x_{1}^{\prime}, x_{2}^{\prime}\right)$, the purchasing threshold level is defined as

$$
Z_{b}^{*}\left(i^{\prime}, x_{2}^{\prime}\right)=\arg \min _{x_{1}}\left\{\left(i^{\prime},\left(x_{1}, x_{2}^{\prime}\right)\right) \mid u_{b}^{*}\left(i^{\prime},\left(x_{1}, x_{2}^{\prime}\right)\right)=0\right\}
$$


where $u_{b}^{*}\left(i^{\prime},\left(x_{1}, x_{2}^{\prime}\right)\right)$ is the optimal purchasing decision in the state $\left(i^{\prime},\left(x_{1}, x_{2}^{\prime}\right)\right)$. The above problem gives us a unique level, $Z_{b}^{*}\left(i^{\prime}, x_{2}^{\prime}\right)$, and it is optimal to buy if the raw materials inventory level for the given state is smaller than $Z_{b}^{*}\left(i^{\prime}, x_{2}^{\prime}\right)$ and to do nothing otherwise.

Q.E.D.

Proof of TheOREM 2: In the proof of Theorem 1, we observe that if an optimal policy does not buy in a state $(i, \underline{\mathbf{x}})$ where $\underline{\mathbf{x}}=\left(x_{1}, x_{2}\right)$, it continues not to buy in all states $(i, \underline{\widehat{\mathbf{x}}})$ where $\underline{\widehat{\mathbf{x}}}=\left(\widehat{x}_{1}, x_{2}\right)$ and $\widehat{x}_{1} \geq x_{1}$. This result directly implies that the optimal purchasing policy for the manufacturer is non-increasing in $x_{1}$.

Let $v^{*}(i, \underline{\mathbf{x}}) \in V$. From Equation (39), we know that if any real-valued function $v^{\prime *}(i, \underline{\mathbf{x}})$ is multimodular with respect to $x_{1}$ and $x_{2}$, then it evidently satisfies the inequalities for supermodularity. So, we have:

$$
v^{\prime *}\left(i, \underline{\mathbf{x}}+e_{1}+e_{2}\right)-v^{\prime *}\left(i, \underline{\mathbf{x}}+e_{2}\right) \geq v^{\prime *}\left(i, \underline{\mathbf{x}}+e_{1}\right)-v^{\prime *}(i, \underline{\mathbf{x}})
$$

If it is optimal not to buy in a state $(i, \underline{\mathbf{x}})$, from Equation $(32)$ we have:

$$
v^{\prime *}\left(i, \underline{\mathbf{x}}+e_{1}\right)-v^{\prime *}(i, \underline{\mathbf{x}})>-c_{i}
$$

Then, we obtain:

$$
v^{\prime *}\left(i, \underline{\mathbf{x}}+e_{1}+e_{2}\right)-v^{\prime *}\left(i, \underline{\mathbf{x}}+e_{2}\right) \geq v^{\prime *}\left(i, \underline{\mathbf{x}}+e_{1}\right)-v^{\prime *}(i, \underline{\mathbf{x}})>-c_{i}
$$

implying that it has to be optimal not to buy in state $\left(i, \underline{\mathbf{x}}+e_{2}\right)$, as well. Therefore, whenever an optimal policy is not to buy in a state $(i, \underline{\mathbf{x}})$ where $\underline{\mathbf{x}}=\left(x_{1}, x_{2}\right)$, it is optimal not to buy in all states $\left(i, \underline{\mathbf{x}}^{\prime \prime}\right)$ where $\underline{\mathbf{x}}^{\prime \prime}=\left(x_{1}, x_{2}^{\prime \prime}\right)$ and $x_{2}^{\prime \prime}>x_{2}$. This result means that the optimal purchasing policy for the manufacturer is non-increasing in $x_{2}$. Specifically, $u_{b}^{*}\left(i,\left(x_{1}, x_{2}\right)\right) \geq u_{b}^{*}\left(i,\left(x_{1}, x_{2}^{\prime \prime}\right)\right)$ holds for any given two states $\left(i,\left(x_{1}, x_{2}\right)\right)$ and $\left(i,\left(x_{1}, x_{2}^{\prime \prime}\right)\right)$ where $x_{2}^{\prime \prime}>x_{2}$. Let $Z_{b}^{*}\left(i, x_{2}\right)$ and $Z_{b}^{\prime *}\left(i, x_{2}^{\prime}\right)$ are the purchasing threshold levels for the states $\left(i,\left(x_{1}, x_{2}\right)\right)$ and $\left(i,\left(x_{1}, x_{2}^{\prime}\right)\right)$ where $x_{2}^{\prime}>x_{2}$. By using the previous result and Equation $(7)$, we can conclude that $Z_{b}^{*}\left(i, x_{2}\right) \geq Z_{b}^{\prime *}\left(i, x_{2}^{\prime}\right)$. This result implies $Z_{b}^{*}\left(i, x_{2}\right)$ that defines the purchasing threshold level for the given state $(i, \underline{\mathbf{x}})$ where $\underline{\mathbf{x}}=\left(x_{1}, x_{2}\right)$ is non-increasing in $x_{2}$.

Q.E.D.

Proof of Theorem 3: Let $v^{\prime *}(i, \underline{\mathbf{x}}) \in V$. Due to Lemma 1 and 2 , we can say that $v^{\prime *}(i, \underline{\mathbf{x}})$ is convex in $\left(e_{2}-e_{1}\right)$, i.e.,

$$
v^{\prime *}\left(i, \underline{\mathbf{x}}-e_{1}+e_{2}\right)-v^{\prime *}(i, \underline{\mathbf{x}}) \geq v^{\prime *}(i, \underline{\mathbf{x}})-v^{\prime *}\left(i, \underline{\mathbf{x}}+e_{1}-e_{2}\right)
$$

If it is optimal to produce in a state $(i, \underline{\mathbf{x}})$, from Equation (33) we have:

$$
v^{\prime}\left(i, \underline{\mathbf{x}}-e_{1}+e_{2}\right)+w<v^{\prime}(i, \underline{\mathbf{x}}) \Longleftrightarrow-w>v^{\prime}\left(i, \underline{\mathbf{x}}-e_{1}+e_{2}\right)-v^{\prime}(i, \underline{\mathbf{x}})
$$


Then, by convexity in $\left(e_{2}-e_{1}\right)$, we have:

$$
-w>v^{* *}\left(i, \underline{\mathbf{x}}-e_{1}+e_{2}\right)-v^{\prime *}(i, \underline{\mathbf{x}}) \geq v^{*}(i, \underline{\mathbf{x}})-v^{* *}\left(i, \underline{\mathbf{x}}+e_{1}-e_{2}\right),
$$

implying that it has to be optimal to produce in state $\left(i, \underline{\mathbf{x}}+e_{1}-e_{2}\right)$, as well. Therefore, whenever an optimal policy is to produce in a state $\left(i,\left(x_{1}, x_{2}\right)\right)$, it is optimal to produce in all states $\left.\left(i, \grave{x}_{1}, \grave{x}_{2}\right)\right)$ where $\grave{x}_{1}>x_{1}$ and $\grave{x}_{2}<x_{1}+x_{2}-\grave{x}_{1}$. A similar discussion can also be adapted to show that if an optimal policy does not produce in a state $\left(i,\left(x_{1}, x_{2}\right)\right)$, it continues not to produce in all states $\left.\left(i, \check{x}_{1}, \check{x}_{2}\right)\right)$ where $\check{x}_{1} \leq x_{1}$ and $\check{x}_{2} \geq x_{1}+x_{2}-\check{x}_{1}$. These statements together mean the existence of an optimal threshold level for the production decision. Specifically, for any given state $\left(i^{\prime},\left(x_{1}^{\prime}, x_{2}^{\prime}\right)\right)$, the purchasing threshold level is defined as

$$
Z_{p}^{*}\left(i^{\prime},\left(x_{1}^{\prime}, x_{2}^{\prime}\right)\right)=\arg \max _{x_{1}}\left\{\left(i^{\prime},\left(x_{1}, x_{1}^{\prime}+x_{2}^{\prime}-x_{1}\right)\right) \mid u_{p}^{*}\left(i,\left(x_{1}, x_{1}^{\prime}+x_{2}^{\prime}-x_{1}\right)\right)=0\right\},
$$

where $u_{p}^{*}\left(i,\left(x_{1}, x_{1}^{\prime}+x_{2}^{\prime}-x_{1}\right)\right)$ is the optimal production decision in state $\left(i,\left(x_{1}, x_{1}^{\prime}+x_{2}^{\prime}-x_{1}\right)\right)$. For any given line $x_{1}^{\prime}+x_{2}^{\prime}=k$ and environment $i^{\prime}$, the above problem gives us a unique level, $Z_{p}^{*}\left(i^{\prime},\left(x_{1}^{\prime}, x_{2}^{\prime}\right)\right)$. That is, it is optimal to produce if $x_{1}^{\prime}>Z_{p}^{*}\left(i^{\prime},\left(x_{1}^{\prime}, x_{2}^{\prime}\right)\right)$ and $x_{2}<k-Z_{p}^{*}\left(i^{\prime},\left(x_{1}^{\prime}, x_{2}^{\prime}\right)\right)$ and to stay idle otherwise. Q.E.D.

Proof of Theorem 4: Let $v^{*}(i, \underline{\mathbf{x}}) \in V$. From Equation (39), we know that if any real-valued function $v^{\prime *}(i, \underline{\mathbf{x}})$ is multimodular with respect to $x_{1}$ and $x_{2}$, then it evidently satisfies the inequalities for superconvexity. So, we have:

$$
v^{\prime *}(i, \underline{\mathbf{x}})+v^{\prime *}\left(i, \underline{\mathbf{x}}+e_{2}\right) \leq v^{\prime *}\left(i, \underline{\mathbf{x}}-e_{1}+e_{2}\right)+v^{\prime *}\left(i, \underline{\mathbf{x}}+e_{1}\right)
$$

By manipulating the above inequality, we obtain:

$$
v^{\prime *}\left(i, \underline{\mathbf{x}}+e_{2}\right)-v^{\prime *}\left(i, \underline{\mathbf{x}}+e_{1}\right) \leq v^{\prime *}\left(i, \underline{\mathbf{x}}-e_{1}+e_{2}\right)-v^{\prime *}(i, \underline{\mathbf{x}}) .
$$

If it is optimal to produce in a state $(i, \underline{\mathbf{x}})$, from Equation (33) we have:

$$
v^{\prime}\left(i, \underline{\mathbf{x}}-e_{1}+e_{2}\right)-v^{\prime}(i, \underline{\mathbf{x}})<-w .
$$

Then, we obtain:

$$
v^{\prime *}\left(i, \underline{\mathbf{x}}+e_{2}\right)-v^{\prime *}\left(i, \underline{\mathbf{x}}+e_{1}\right) \leq v^{\prime *}\left(i, \underline{\mathbf{x}}-e_{1}+e_{2}\right)-v^{\prime *}(i, \underline{\mathbf{x}})<-w,
$$

implying that it has to be optimal to produce in state $\left(i, \underline{\mathbf{x}}+e_{1}\right)$, as well. Therefore, whenever an optimal policy is to produce in a state $(i, \underline{\mathbf{x}})$ where $\underline{\mathbf{x}}=\left(x_{1}, x_{2}\right)$, it is optimal to produce in all states $(i, \underline{\underline{\mathbf{x}}})$ where $\underline{\mathbf{x}}=\left(\grave{x}_{1}, x_{2}\right)$ and $\grave{x_{1}}>x_{1}$. This result means that the optimal production policy for the manufacturer is 
non-decreasing in $x_{1}$. Specifically, $u_{p}^{*}\left(i,\left(x_{1}, x_{2}\right)\right) \leq u_{p}^{*}\left(i,\left(\grave{x}_{1}, x_{2}\right)\right)$ holds for any given two states $\left(i,\left(x_{1}, x_{2}\right)\right)$ and $\left.\left(i, \grave{x}_{1}, x_{2}\right)\right)$ where $\grave{x_{1}}>x_{1}$. A similar analysis can also be adapted to prove that the optimal production policy for the manufacturer is non-increasing in $x_{2}$. Let $Z_{p}^{*}\left(i,\left(x_{1}, x_{2}\right)\right)$ and $Z_{p}^{\prime *}\left(i,\left(x_{1}, \check{x}_{2}\right)\right)$ are the production threshold levels for the states $\left(i,\left(x_{1}, x_{2}\right)\right)$ and $\left(i,\left(x_{1}, \check{x}_{2}\right)\right)$ where $\check{x}_{2}>x_{2}$. By using the previous results and Equation (8), we can conclude that $Z_{p}^{*}\left(i,\left(x_{1}, x_{2}\right)\right) \leq Z_{p}^{\prime *}\left(i,\left(x_{1}, x_{2}\right)\right)$. This result implies $Z_{p}^{*}\left(i,\left(x_{1}, x_{2}\right)\right)$ that defines the production threshold level for the given state $(i, \underline{\mathbf{x}})$ where $\underline{\mathbf{x}}=\left(x_{1}, x_{2}\right)$ is non-increasing in $x_{2}$. Q.E.D.

Proof of Theorem 5: Let $v^{\prime *}(i, \underline{\mathbf{x}}) \in V$. Due to Lemma 1, we can say that $v^{\prime *}(i, \underline{\mathbf{x}})$ is convex with respect to $x_{2}$ for each $i$ and $x_{1}$, i.e.,

$$
v^{\prime *}\left(i, \underline{\mathbf{x}}+e_{2}\right)-v^{\prime *}(i, \underline{\mathbf{x}}) \geq v^{\prime}(i, \underline{\mathbf{x}})-v^{\prime *}\left(i, \underline{\mathbf{x}}-e_{2}\right) .
$$

If it is optimal to sell in a state $(i, \underline{\mathbf{x}})$, from Equation $(35)$ we have:

$$
v^{\prime *}\left(i, \underline{\mathbf{x}}-e_{2}\right)-s_{i}<v^{\prime *}(i, \underline{\mathbf{x}}) \Longleftrightarrow-s_{i}<v^{\prime *}(i, \underline{\mathbf{x}})-v^{\prime *}\left(i, \underline{\mathbf{x}}-e_{2}\right)
$$

Then, by convexity, we have:

$$
v^{\prime *}\left(i, \underline{\mathbf{x}}+e_{2}\right)-v^{\prime *}(i, \underline{\mathbf{x}}) \geq v^{\prime *}(i, \underline{\mathbf{x}})-v^{\prime *}\left(i, \underline{\mathbf{x}}-e_{2}\right)>-s_{i}
$$

meaning that it has to be optimal to sell in state $\left(i, \underline{\mathbf{x}}+e_{2}\right)$, as well. Hence, whenever an optimal policy is to sell in a state $(i, \underline{\mathbf{x}})$ where $\underline{\mathbf{x}}=\left(x_{1}, x_{2}\right)$, it is optimal to sell in all states $(i, \underline{\dot{\mathbf{x}}})$ where $\underline{\dot{\mathbf{x}}}=\left(x_{1}, \dot{x}_{2}\right)$ and $\dot{x}_{2}>x_{2}$. For a fixed $i$ and $x_{1}$, a similar discussion can also be adapted to show that if it is not optimal to sell in a certain state, it continues not to sell in all states that have a lower level of the finished goods inventory. These two statements together imply the existence of an optimal threshold level for the sales decision. Specifically, for any given state $\left(i^{\prime}, \underline{\mathbf{x}}^{\prime}\right)$ where $\left(x_{1}^{\prime}, x_{2}^{\prime}\right)$, the sales threshold level is defined as

$$
Z_{r}^{*}\left(i^{\prime}, x_{1}^{\prime}\right)=\arg \min _{x_{2}}\left\{\left(i^{\prime},\left(x_{1}^{\prime}, x_{2}\right)\right) \mid u_{r}^{*}\left(i^{\prime},\left(x_{1}^{\prime}, x_{2}\right)\right)=1\right\}
$$

where $u_{r}^{*}\left(i^{\prime},\left(x_{1}^{\prime}, x_{2}\right)\right)$ is the optimal sales decision in state $\left(i^{\prime},\left(x_{1}^{\prime}, x_{2}\right)\right)$. The above problem gives us a unique level, $Z_{r}^{*}\left(i^{\prime}, x_{1}^{\prime}\right)$, and it is optimal to sell if the level of the finished goods inventory is greater than $Z_{r}^{*}\left(i^{\prime}, x_{1}^{\prime}\right)$ and not to sell otherwise.

Q.E.D.

Proof of Theorem 6: In the proof of Theorem 5, we observe that whenever an optimal policy is to sell in a state $(i, \underline{\mathbf{x}})$ where $\underline{\mathbf{x}}=\left(x_{1}, x_{2}\right)$, it is optimal to sell in all states $(i, \underline{\dot{\mathbf{x}}})$ where $\underline{\dot{\mathbf{x}}}=\left(x_{1}, \dot{x}_{2}\right)$ and $\dot{x}_{2}>x_{2}$. This result directly implies that the optimal sales policy for the manufacturer is non-increasing in $x_{2}$. 
Let $v^{\prime *}(i, \underline{\mathbf{x}}) \in V$. From Equation (39), we know that if any real-valued function $v^{\prime *}(i, \underline{\mathbf{x}})$ is multimodular with respect to $x_{1}$ and $x_{2}$, then it evidently satisfies the inequalities for supermodularity. So, we have:

$$
v^{\prime *}\left(i, \underline{\mathbf{x}}+e_{1}-e_{2}\right)-v^{\prime *}\left(i, \underline{\mathbf{x}}+e_{1}\right) \leq v^{* *}\left(i, \underline{\mathbf{x}}-e_{2}\right)-v^{\prime *}(i, \underline{\mathbf{x}}) .
$$

If it is optimal to sell in a state $(i, \underline{\mathbf{x}})$, from Equation (34) we have:

$$
v^{\prime *}\left(i, \underline{\mathbf{x}}-e_{2}\right)-v^{\prime *}(i, \underline{\mathbf{x}})<s_{i} .
$$

Then, we obtain:

$$
v^{\prime *}\left(i, \underline{\mathbf{x}}+e_{1}-e_{2}\right)-v^{\prime *}\left(i, \underline{\mathbf{x}}+e_{1}\right) \leq v^{\prime *}\left(i, \underline{\mathbf{x}}-e_{2}\right)-v^{\prime *}(i, \underline{\mathbf{x}})<s_{i},
$$

implying that it has to be optimal to sell in state $\left(i, \underline{\mathbf{x}}+e_{1}\right)$, as well. Therefore, whenever an optimal policy is to sell in a state $(i, \underline{\mathbf{x}})$ where $\underline{\mathbf{x}}=\left(x_{1}, x_{2}\right)$, it is optimal to sell in all states $(i, \underline{\ddot{x}})$ where $\underline{\ddot{x}}=\left(\ddot{x}_{1}, x_{2}\right)$ and $\ddot{x}_{1}>x_{1}$. This result means that the optimal sales policy for the manufacturer is non-decreasing in $x_{1}$. Specifically, $u_{r}^{*}\left(i,\left(x_{1}, x_{2}\right)\right) \leq u_{r}^{*}\left(i,\left(\ddot{x}_{1}, x_{2}\right)\right)$ holds for any given two states $\left(i,\left(x_{1}, x_{2}\right)\right)$ and $\left(i,\left(\ddot{x}_{1}, x_{2}\right)\right)$ where $\ddot{x}_{1}>x_{1}$. Let $Z_{r}^{*}\left(i, x_{1}\right)$ and $Z_{r}^{\prime *}\left(i, \ddot{x}_{1}\right)$ are the sales threshold levels for the states $\left(i,\left(x_{1}, x_{2}\right)\right)$ and $\left(i,\left(\ddot{x}_{1}, x_{2}\right)\right)$ where $\ddot{x}_{1}>x_{1}$. By using the previous result and Equation (9), we can conclude that $Z_{r}^{*}\left(i, x_{1}\right) \geq Z_{r}^{\prime *}\left(i, \ddot{x}_{1}\right)$. This result implies $Z_{r}^{*}\left(i, x_{1}\right)$ that defines the sales threshold level for the given state $(i, \underline{\mathbf{x}})$ where $\underline{\mathbf{x}}=\left(x_{1}, x_{2}\right)$ is non-increasing in $x_{1}$.

Q.E.D.

\section{Appendix B:}

In this section, we introduce the procedure forming the transition matrices that are necessary to obtain the specified correlation coefficients between sales and purchasing prices. In the procedure, we suppose that $\mathrm{E}[C]$, $\operatorname{Var}[C], \mathrm{E}[S], \operatorname{Var}[S], \rho_{C S}, s_{H}, s_{L}, c_{H}$, and $c_{L}$ are given as the input parameters. Additionally, the average time spent in each environment state is given. For the environment state $i$, it is denoted by $T_{i}$.

In the first step, by using the parameters $\mathrm{E}[C], \operatorname{Var}[C], \mathrm{E}[S], \operatorname{Var}[S], \rho_{C S}, s_{H}, s_{L}, c_{H}$, and $c_{L}$, we construct and solve the following linear equation system:

$$
\left[\begin{array}{c}
\mathrm{E}[S] \\
\mathrm{E}[C] \\
\rho_{C S} \sqrt{\operatorname{Var}[C] \operatorname{Var}[S]}+\mathrm{E}[C] \mathrm{E}[S] \\
1
\end{array}\right]=\left[\begin{array}{cccc}
s_{H} & s_{H} & s_{L} & s_{L} \\
c_{H} & c_{L} & c_{H} & c_{L} \\
s_{H} c_{H} & c_{L} s_{H} & c_{H} s_{L} & c_{L} s_{L} \\
1 & 1 & 1 & 1
\end{array}\right]\left[\begin{array}{c}
\pi_{1} \\
\pi_{2} \\
\pi_{3} \\
\pi_{4}
\end{array}\right] .
$$






Figure 9 Illustration for the structure of the transition matrix considered in the numerical examples

The solution of the above equation system yields the long-run probabilities of being in each environment state. It has a unique solution for the given parameters as far as $s_{H} \neq s_{L}$ and $c_{H} \neq c_{L}$ hold.

In the second step, we substitute the solution of the above equation system into Equation (22). With this effort, we can define a transition matrix ensuring that $\mathrm{E}[C], \operatorname{Var}[C], \mathrm{E}[S], \operatorname{Var}[S]$, and $\rho_{C S}$ are the same as given at the beginning of the procedure. Since the time between each transition is exponentially distributed, the transition matrix should not allow the sales and purchasing prices to change at the same time. Therefore, the transition matrix always preserves the structure illustrated in the transition diagram in Figure (9). Specifically, it implies that $q_{23}=q_{32}=q_{14}=q_{41}=0$ in all the numerical instances. The exponentially distributed transition times also imply that the average time spent in environment state $i$ can be defined as $T_{i}=\frac{1}{q_{i i}}$ where $q_{i i}=\sum_{j \in \mathbb{E} \backslash\{i\}} q_{i j}$. This relationship allows us to characterize the diagonal elements of the transition matrix since the average time spent in each environment state is already given. By considering these facts, we simplify the equation system obtained with Equation (22) as follows:

$$
\begin{aligned}
& \frac{\pi_{2} q_{21}}{\pi_{1}}+\frac{\pi_{3} q_{31}}{\pi_{1}}=\frac{1}{T_{1}}, \\
& \frac{\pi_{1} q_{12}}{\pi_{2}}+\frac{\pi_{4} q_{42}}{\pi_{2}}=\frac{1}{T_{2}}, \\
& \frac{\pi_{1} q_{13}}{\pi_{3}}+\frac{\pi_{4} q_{43}}{\pi_{3}}=\frac{1}{T_{3}}, \\
& \frac{\pi_{2} q_{24}}{\pi_{4}}+\frac{\pi_{3} q_{34}}{\pi_{4}}=\frac{1}{T_{4}} .
\end{aligned}
$$

Let suppose that $q_{12}$ and $q_{21}$ equal $a$ and $b$, respectively. Since the sum of the elements in a row of the transition matrix equals zero, $q_{13}$ is $1 / T_{1}-a$ and $q_{24}$ is $1 / T_{2}-b$. By substituting $q_{21}=b$ into Equation (63), we define the transition rate from state 3 to 1 as $q_{31}=\left(\pi_{1}-b T_{1} \pi_{2}\right) / T_{1} \pi_{3}$. Replacing $q_{12}=a$ into Equation (64) yields the transition rate from state 4 to 2 , that is, $q_{42}=\left(\pi_{2}-a \pi_{1} T_{2}\right) / T_{2} \pi_{4}$. The values of $q_{13}$ and $q_{24}$ are also substituted into Equations (65) and (66), respectively. With this effort, we find that 
$q_{34}=\left(T_{2} \pi_{4}-T_{4} \pi_{2}\right) / T_{1} T_{4} \pi_{3}+\pi_{2} b / \pi_{3}$ and $q_{43}=\left(T_{1} \pi_{3}-T_{3} \pi_{1}\right) / T_{1} T_{3} \pi_{4}+\pi_{1} a / \pi_{4}$. So, for the given $a$ and $b$ values, the solution of the above equation system is expressed as follows:

$$
Q=\left[\begin{array}{cccc}
-\frac{1}{T_{1}} & a & \frac{1}{T_{1}}-a & 0 \\
b & -\frac{1}{T_{2}} & 0 & \frac{1}{T_{2}}-b \\
\frac{\pi_{1}-b T_{1} \pi_{2}}{T_{1} \pi_{3}} & 0 & -\frac{1}{T_{3}} & \frac{T_{2} \pi_{4}-T_{4} \pi_{2}}{T_{1} T_{4} \pi_{3}}+\frac{\pi_{2} b}{\pi_{3}} \\
0 & \frac{\pi_{2}-a \pi_{1} T_{2}}{T_{2} \pi_{4}} & \frac{T_{1} \pi_{3}-T_{3} \pi_{1}}{T_{1} T_{3} \pi_{4}}+\frac{\pi_{1} a}{\pi_{4}} & -\frac{1}{T_{4}}
\end{array}\right] .
$$

As a transition matrix, the off-diagonal elements of $Q$ should be non-negative and the sum of each row should be zero. These facts respectively imply the following conditions on $a, b$, and $T_{i}$ :

$$
\begin{gathered}
a \in\left(\max \left\{0, \frac{T_{1} \pi_{3}-T_{3} \pi_{1}}{\pi_{1} T_{1} T_{3}}\right\}, \min \left\{\frac{1}{T_{1}}, \frac{\pi_{2}}{\pi_{1} T_{2}}\right\}\right), \\
b \in\left(\max \left\{0, \frac{T_{4} \pi_{2}-T_{2} \pi_{4}}{\pi_{2} T_{1} T_{4}}\right\}, \min \left\{\frac{1}{T_{2}}, \frac{\pi_{1}}{\pi_{2} T_{1}}\right\}\right), \\
\frac{\pi_{1}}{T_{1}}+\frac{\pi_{4}}{T_{4}}=\frac{\pi_{2}}{T_{2}}+\frac{\pi_{3}}{T_{3}} \text { and } T_{i}>0, \text { where } i \in\{1,2,3,4\} .
\end{gathered}
$$

As far as the above conditions are satisfied for the input parameters, the matrix defined in Equation (67) will be a proper transition matrix ensuring that $\mathrm{E}[C], \operatorname{Var}[C], \mathrm{E}[S], \operatorname{Var}[S]$, and $\rho_{C S}$ are the same as given at the beginning of the procedure.

We follow the above-mentioned procedure to form the transition matrices that are used to obtain the specified correlation coefficients between sales and purchasing prices. All the input parameters used to initiate this procedure are specified at the beginning of each subsection within the Numerical Analysis section. For each given correlation coefficient, we construct the equation system defined in Equation (62) and solve it. While forming the equation system for the specified correlation coefficients, we keep the expectations and variances of the sales and purchasing prices at the same levels for the numerical examples to be examined in the same group. Hence, we avoid the case in which the expectations and variances of the sales and purchasing prices change each time the correlation coefficient changes. Subsequently, for each correlation coefficient, we substitute the obtained solution into Equation (67) and define the corresponding transition matrix. While defining the transition matrices for the specified correlation coefficients, we consider that $T_{1}=T_{4}, T_{2}=T_{3}$, $a=1 / 2 T_{1}$, and $b=1 / 2 T_{2}$. This setting allows the conditions defined in Equations (68), (69), and (70) to be 
immediately satisfied. As a result of this effort, we define the transition matrices so as to give the desired correlation coefficients.

\section{References}

Altman, E., B. Gaujal, and A. Hordijk (2000). Multimodularity, convexity, and optimization properties. Mathematics of Operations Research 25(2), 324-347.

Arnold, J., S. Minner, and B. Eidam (2009). Raw material procurement with fluctuating prices. International Journal of Production Economics 121(2), 353-364.

Arnold, J., S. Minner, and M. Morrocu (2011). A continuous time dynamic optimal control manufacturing problem. International Journal of Inventory Research 1(3-4), 248-261.

Berling, P. and V. Martínez-de Albéniz (2011). Optimal inventory policies when purchase price and demand are stochastic. Operations Research 59(1), 109-124.

Berling, P. and Z. Xie (2014). Approximation algorithms for optimal purchase/inventory policy when purchase price and demand are stochastic. OR Spectrum 36(4), 1077-1095.

Bertsekas, D. P. (1995). Dynamic programming and optimal control, Volume 2. Athena Scientific Belmont.

Cao, X., C. Huang, and H. Wen (2009). Acquisition and production management with two inventories under fluctuating prices and demand. Wuhan University Journal of Natural Sciences 14(4), 293-298.

De Cuypere, E., K. De Turck, H. Bruneel, and D. Fiems (2013). Optimal inventory management in a fluctuating market. In A. Dudin and K. De Turck (Eds.), Lecture Notes in Computer Science, Volume 7984, pp. 158-170. Springer-Verlag.

Erdem, A. S. and S. Özekici (2002). Inventory models with random yield in a random environment. International Journal of Production Economics 78(3), 239-253.

Gallego, G. and H. Hu (2004). Optimal policies for production/inventory systems with finite capacity and markov-modulated demand and supply processes. Annals of Operations Research 126(1), 21-41.

Gavirneni, S. (2004). Periodic review inventory control with fluctuating purchasing costs. Operations Research Letters 32(4), 374-379.

Gayon, J. P., I. Talay Değirmenci, F. Karaesmen, and E. L. Örmeci (2009). Optimal pricing and production policies of a make-to-stock system with fluctuating demand. Probability in the Engineering and Informational Sciences 23(2), 205-230.

Koole, G. (1998). Structural results for the control of queueing systems using event-based dynamic programming. Queueing systems 30(3-4), 323-339. 
Koole, G. (2004). Convexity in tandem queues. Probability in the Engineering and Informational Sciences 18(1), 13-31.

Lippman, S. A. (1975). Applying a new device in the optimization of exponential queuing systems. Operations Research 23(4), 687-710.

Liu, Y. and J. Yang (2015). Joint pricing-procurement control under fluctuating raw material costs. International Journal of Production Economics 168, 91-104.

Mohebbi, E. (2006). A production-inventory model with randomly changing environmental conditions. European Journal of Operational Research 174(1), 539-552.

Nadar, E., M. Akan, and A. Scheller-Wolf (2014). Optimal structural results for assemble-to-order generalized M-systems. Operations Research 62(3), 571-579.

Özekici, S. and M. Parlar (1999). Inventory models with unreliable suppliers in a random environment. Annals of Operations Research 91, 123-136.

Papachristos, S. and A. Katsaros (2008). A periodic-review inventory model in a fluctuating environment. IIE Transactions 40(3), 356-366.

Puterman, M. L. (2014). Markov decision processes: Discrete stochastic dynamic programming. John Wiley \& Sons.

Wu, O. Q. and H. Chen (2010). Optimal control and equilibrium behavior of production/inventory systems. Management Science 56(8), 1362-1379. 